\title{
An effect of extra compactified dimensions of space in the molecule $\mathrm{NO}_{2}$
}

\author{
Hans-Georg Weber $^{1,2, a}{ }_{(\mathbb{D}}$ \\ ${ }^{1}$ Fraunhofer Institute for Telecommunications, Heinrich-Hertz-Institut, Einsteinufer 37, 10587 Berlin, \\ Germany \\ 2 Present address: Sudetenstr.16, 63916 Amorbach, Germany
}

Received 23 March 2021 / Accepted 23 November 2021 / Published online 28 December 2021 (C) The Author(s) 2021

\begin{abstract}
The theory of large extra compactified dimensions of space (ADD-model) predicts that gravity may become strong in a compactification space of the size of a molecule and may affect the vibrational motion of a molecule. In triatomic molecules like $\mathrm{NO}_{2}$ nuclear dynamics is strongly coupled to electronic dynamics at the intersection of electronic states (conical intersection). We discuss experimental results on $\mathrm{NO}_{2}$ which reveal that the collision-free molecule optically excited into a symmetric stretch vibration mode of an electronic state with conical intersection undergoes an irreversible non-radiative transition into an asymmetric stretch vibration mode in combination with a change of the electronic state. We suggest ascribing this irreversible non-radiative transition to a gravitational perturbation on the vibrational motion in $\mathrm{NO}_{2}$. This gravitational perturbation deactivates the upper state of the optical transition. The width of the absorption line is given by the characteristic time of the gravitational perturbation and not by the radiative lifetime of the excited molecular state.
\end{abstract}

\section{Introduction}

The structure of a molecule is commonly described in the 3-dimensional space we see. However, modern physics (string theory) requires six or seven extra spatial dimensions (e.g. $[1,2]$ ). In the theoretical frameworks with extra dimensions, our observed 3 -dimensional space is merely a subspace (a brane) embedded in a higher dimensional space with $3+n$ dimensions where $\mathrm{n}$ is the number of extra spatial dimensions. The three forces of the standard model are confined to the brane but the gravitational field spreads throughout the full $3+\mathrm{n}$ spatial volume. Moreover, the extra dimensional space must be compactified, to avoid a contradiction to Newtonian gravity. For a long time, it was assumed that extra dimensions have a compactification radius $\mathrm{R}_{\mathrm{c}}$ about the order of the Planck length $\left(\mathrm{R}_{\mathrm{c}} \approx 10^{-33} \mathrm{~cm}\right)$. However, the work of Arkani-Hamed, Dimopoulos, and Dvali (ADD) on large extra compactified dimensions of space changed the view on the extra spatial dimensions [3]. The physics of large extra dimensions offers new insights and solutions to fundamental questions in the standard model (e.g. [3-8]).

The ADD-model predicts that gravity may become strong in a compactification space of the size of a molecule and may affect the vibrational motion. Outside the compactification radius $R_{c}$ gravity follows the 3-dimensional Newtonian law, but for $\mathrm{r}<\mathrm{R}_{\mathrm{c}}$ the extra-

\footnotetext{
a e-mail: hans-georg.weber@gmx.de (corresponding author)
}

dimensional gravitational potential of the ADD- model is effective. In Refs. $[9,10]$, the contribution of the gravitational potential to the splitting of vibrational levels is analysed for diatomic molecules (neutral and ionic hydrogen molecules and the deuterated isotopomers) by combining precision measurements and calculations (including relativistic and quantum electrodynamical effects), and the upper bound $\mathrm{R}_{\mathrm{c}}<0.6 \mu \mathrm{m}$ is derived for the case of $\mathrm{n}=7$ extra dimensions.

We apply the ADD-model to the molecule $\mathrm{NO}_{2}$ [11]. Triatomic molecules like $\mathrm{NO}_{2}$ are interesting candidates for a study of the extra-dimensional gravitational potential suggested by the ADD- model, because these molecules are the simplest systems in which electronic dynamics is strongly coupled to nuclear dynamics (e.g.[12-16] and references given there). In the optically excited $\mathrm{NO}_{2}$, the motion of the nuclei is not strictly bound to the potential energy surface (PES) of a single electronic state as in diatomic molecules. In the optically excited $\mathrm{NO}_{2}$, several electronic states may intersect (conical intersection) enabling transitions between these electronic states. For example, laser light $\left(\lambda_{\text {ex }} \approx 600 \mathrm{~nm}\right)$ excites $\mathrm{NO}_{2}$ molecules from the electronic ground state $\mathrm{X}^{2} \mathrm{~A}_{1}$ to the excited electronic state $\mathrm{A}^{2} \mathrm{~B}_{2}$ ([17] and references given there). At this excitation energy, there are also high lying vibrational levels of the electronic ground state $\mathrm{X}^{2} \mathrm{~A}_{1}$. The two potential energy surfaces of the electronic states $\mathrm{A}^{2} \mathrm{~B}_{2}$ and $\mathrm{X}^{2} \mathrm{~A}_{1}$ intersect at a particular bond angle that depends on the bond length. At this intersection the nuclei of the 
molecule being in the symmetric stretch and bending vibration mode in the state $\mathrm{A}^{2} \mathrm{~B}_{2}$ may pass over into the asymmetric stretch vibration mode in the state $\mathrm{X}$ ${ }^{2} \mathrm{~A}_{1}$. Parity is conserved in this process.

The presently accepted model describing the properties of the optically excited $\mathrm{NO}_{2}$ is the model of interelectronic level mixing $[18,19]$. This model assumes that the vibronic (electronic and vibrational) coupling of low vibrational levels of the state $\mathrm{A}^{2} \mathrm{~B}_{2}$ with high vibrational levels of the state $\mathrm{X}^{2} \mathrm{~A}_{1}$ is a reversible process causing a mixing of vibrational levels of the two electronic states to eigenstates of the molecule. An example of this interelectronic level mixing is the theory of the "anomalously long radiative lifetimes" of $\mathrm{NO}_{2}[18,19]$. The radiative lifetimes $\tau_{R}$ of the optically excited states are about by a factor of 10 larger than the lifetimes $\tau_{\text {in }}$ calculated from the integrated absorption coefficient $[18,20]$. As the eigenstates of the molecule have both $\mathrm{A}^{2} \mathrm{~B}_{2}$ and $\mathrm{X}^{2} \mathrm{~A}_{1}$ character, the $\mathrm{X}^{2} \mathrm{~A}_{1}$ character in the molecular eigenstate is responsible for the long radiative lifetimes whereas a strong optical transition moment from the ground state to the state $\mathrm{A}^{2} \mathrm{~B}_{2}$ is responsible for the small value of $\tau_{\text {in }}$.

Below we report that the application of the ADDmodel on $\mathrm{NO}_{2}$ gives a different explanation for the discrepancy of the values for $\tau_{R}$ and $\tau_{i n}$. The ADDmodel implies that the collision-free $\mathrm{NO}_{2}$ is not isolated. It is always coupled to the extra compactified dimensions of space via the gravitational interaction in the extra compactified dimensions. For this reason, we introduced the model of the space-coupled molecule in Ref. [11]. Contrary to the model of interelectronic level mixing in which the vibronic coupling leads to molecular eigenstates comprising a mixture of $\mathrm{A}^{2} \mathrm{~B}_{2}$ and $\mathrm{X}^{2} \mathrm{~A}_{1}$ characters, the model of the space-coupled molecule implies that gravitational perturbation on the vibrational motion causes an irreversible vibronic transition of the molecule from the symmetric stretch and bending vibration mode in the state $\mathrm{A}^{2} \mathrm{~B}_{2}$ to the asymmetric stretch vibration mode in the state $\mathrm{X}^{2} \mathrm{~A}_{1}$. We will show that the experimental results support the model of the space-coupled molecule.

We discuss the model of the space-coupled molecule considering the following experimental situation. $\mathrm{NO}_{2}$ is optically excited from a state $\mid \mathrm{g}>$ of the electronic ground state $\mathrm{X}^{2} \mathrm{~A}_{1}$ to a state $\mid \mathrm{e}>$ near the conical intersection of the electronic states $\mathrm{A}^{2} \mathrm{~B}_{2}$ and $\mathrm{X}^{2} \mathrm{~A}_{1}$. Based on the model of the space-coupled molecule we assume that the molecule is confined either to one or to two compactification spaces. Thus, we introduce $\mid \mathrm{g}, 1>$ and $\mid \mathrm{g}, 2>$ as well as $\mid \mathrm{e}, 1>$ and $\mid \mathrm{e}, 2>$ as states of the space-coupled molecule. Here " 1 " and "2" indicate that the space-coupled molecule is confined to a single compactification space in $\mid \mathrm{g}, 1>$ and $\mid \mathrm{e}, 1>$ and to two separate compactification spaces in $\mid \mathrm{g}, 2>$ and $\mid \mathrm{e}, 2>$. We will further assume that optical transitions conserve the property " 1 " or " 2 " of the space-coupled molecule, because optical transitions have no effect on the extra compactified dimensions of space. We assign the state $\mid \mathrm{g}, 1>$ to the molecule in the totally symmetric ground vibrational level $(0,0,0)$ in the electronic ground state $\mathrm{X}^{2} \mathrm{~A}_{1}$ and $\mid \mathrm{e}, 1>$ to the molecule in the excited symmetric stretch vibration mode in the electronic state $\mathrm{A}^{2} \mathrm{~B}_{2}$ in which $\mathrm{NO}_{2}$ has a larger $\mathrm{N}-\mathrm{O}$ bond length than in $\mid \mathrm{g}, 1>[21,22]$. In $\mid \mathrm{e}, 2>$ the molecule vibrates in the asymmetric stretch vibration mode in the electronic state $\mathrm{X}^{2} \mathrm{~A}_{1}$ having a short and a long $\mathrm{N}-\mathrm{O}$ bond length with the two nuclei of the short N$\mathrm{O}$ bond length being confined to one compactification space whereas the $\mathrm{O}$ nucleus of the long $\mathrm{N}-\mathrm{O}$ bond length is confined to a separate compactification space. Gravitational perturbation on the vibrational motion in $\mid \mathrm{e}, 1>$ causes the transition $|\mathrm{e}, 1>\rightarrow| \mathrm{e}, 2>$. The consistency of the model requires an optical transition between $\mid \mathrm{g}, 2>$ and $\mid \mathrm{e}, 2>$. However, $\mid \mathrm{g}, 2>$ is different from $|\mathrm{g}, 1>,| \mathrm{e}, 1>$ and $\mid \mathrm{e}, 2>$ which may be stationary states of the molecule in the absence of gravitational interaction. But $\mid \mathrm{g}, 2>$ cannot be a stationary state, because the electronic ground state of the molecule has only a single PES, which allows only a single totally symmetric ground vibrational level $(0,0,0)$. Therefore, we assume that the lifetime of the molecule in $\mid \mathrm{g}, 2>$ is limited by the transition $|\mathrm{g}, 2>\rightarrow| \mathrm{g}, 1>$ to a few vibrational periods. This transition is not caused by gravitational perturbation on the vibrational motion but by the electric force of the PES in the ground state $\mathrm{X}^{2} \mathrm{~A}_{1}$. Consequently, optical excitation of the molecule starts always from $\mid \mathrm{g}, 1>$. The following equations compile the considered model of the space-coupled molecule.

$$
\begin{aligned}
& \text { states of the space-coupled molecule } \\
& \quad\{|\mathrm{g}, 1>,| \mathrm{g}, 2>\}, \quad\{|\mathrm{e}, 1>,| \mathrm{e}, 2>\} \\
& \text { optical transitions }
\end{aligned}
$$$$
|\mathrm{g}, 1>\leftrightarrow| \mathrm{e}, 1>, \quad|\mathrm{g}, 2>\leftrightarrow| \mathrm{e}, 2>
$$

non-radiative transitions

$$
|\mathrm{g}, 1>\leftarrow| \mathrm{g}, 2>, \quad|\mathrm{e}, 1>\rightarrow| \mathrm{e}, 2>
$$

The transition $|\mathrm{e}, 1>\rightarrow| \mathrm{e}, 2>$ of the molecule from the symmetric stretch and bending vibration mode in the state $\mathrm{A}^{2} \mathrm{~B}_{2}$ to the asymmetric stretch vibration mode in the state $\mathrm{X}^{2} \mathrm{~A}_{1}$ is caused by gravitational perturbation on the vibrational motion in $\mid e, 1>$. The nuclei of $\mathrm{NO}_{2}$ see additionally to the usual potential energy surface (PES) of the electronic state also the extra-dimensional gravitational potential suggested by ADD-theory (gravitational PES). If the two PESs do not superimpose symmetrically, we expect an asymmetric perturbation on the symmetric vibrational motion of the molecule. In Ref. [11] we propose an asymmetric perturbation caused by a non-rigid compactification space having a shape, which is fluctuating in time with the characteristic time constant $\tau_{0}$. This perturbation induces randomly asymmetry into the vibrational motion with an average repetition frequency $\left(1 / \tau_{0}\right)$. The transition $|\mathrm{e}, 1>\rightarrow| \mathrm{e}, 2>$ is irreversible in contradiction to the model of interelectronic level mixing. The effect of the gravitational perturbation on the vibrational motion is different in $\mid e, 1>$ and $\mid e, 2>$ because the shape of the molecule is different in the two states. 
The gravitational perturbation on the vibrational motion in the state $\mid \mathrm{e}, 1>$ affects the absorption properties of the optical transition $|\mathrm{g}, 1>\leftrightarrow| \mathrm{e}, 1>$ as we show in Ref. [11]. The upper state of the optical transition $|\mathrm{g}, 1>\leftrightarrow| \mathrm{e}, 1>$ is deactivated by the gravitational perturbation on the vibrational motion and not by spontaneous emission [11]. The width of the absorption line is given by the characteristic time of the gravitational perturbation and not by the radiative lifetime of the excited molecule. Due to the gravitational perturbation, the vibration in $\mid \mathrm{e}, 1>$ does not comprise a single frequency $\tilde{\omega}_{0}$ representing an unlimited duration of free oscillation, but it comprises a band of frequencies with the probability distribution $\mathrm{W}\left(\tilde{\omega}-\tilde{\omega}_{0}\right) \sim\left[1+\left(\tilde{\omega}-\tilde{\omega}_{0}\right)^{2}\left(\tau_{0}\right)^{2}\right]^{-1}$ centred at the frequency $\tilde{\omega}_{0}$ and with the time constant $\tau_{0}$ as mean time between two perturbations [11]. The result of the gravitational perturbation is a line broadening of the optical transition $|\mathrm{g}, 1>\leftrightarrow| \mathrm{e}, 1>$. The optical resonance frequencies are distributed over a band having the width (FWHM) $\Delta \omega=2\left(\tau_{0}\right)^{-1}$ or $\Delta \nu=\left(\pi \tau_{0}\right)^{-1}$. The time constant $\tau_{0}$ is expected to agree with the lifetime $\tau_{\text {in }}$ evaluated from measurements of the integrated absorption coefficient giving values for $\tau_{\text {in }}$ between $1 \mu$ s and $4 \mu \mathrm{s}([18,20]$ and references given there). According to theory, $\tau_{\text {in }}$ is the characteristic time of the mechanism that deactivates the upper state of the optical transition and $\tau_{\text {in }}=\tau_{R}$ is expected if spontaneous emission is the only mechanism of deactivation of an upper state [23]. As we will report below, the experiments reveal $\tau_{0} \approx 3 \mu \mathrm{s}$ in good agreement with the values for $\tau_{\text {in }}$.

In Ref.[11], we used the misleading formulation "substructure underlying the expected eigenstate $\mid e>$ of the molecule" for the states $\mid \mathrm{e}, 1>$ and $\mid \mathrm{e}, 2>$. The correct formulation is "the expected eigenstate $\mid e>$ in the model of interelectronic level mixing is replaced by the states $\mid \mathrm{e}, 1>$ and $\mid \mathrm{e}, 2>$ with the gravity induced irreversible transition $|\mathrm{e}, 1>\rightarrow| \mathrm{e}, 2>$ in the model of the space-coupled molecule".

In Sects. 2-6, we report on experimental work on the molecule $\mathrm{NO}_{2}$. The results of these experiments yield expected molecular properties as radiative lifetimes and g-factors [24-26], but also unusual results which are not seen in similar experiments on atoms and diatomic molecules. An unusual result is the "inversion effect" ([27] and Sect. 3). All efforts to explain this effect conventionally failed ([28] and references given there). The description of this effect requires the assumption of an irreversible non-radiative transition $\mid \mathrm{e}, 1>\rightarrow \mathrm{e}, 2>$ in the collision-free molecule. Another unusual result is the observation of two characteristic times $\tau_{\mathrm{R}} \approx 35 \mu \mathrm{s}$ and $\tau_{0} \approx 3 \mu$ s associated with each hyperfine structure (hfs)-level of the optically excited molecule. $\tau_{\mathrm{R}}$ is measured as single exponential radiative decay time in time resolved fluorescence decay measurements $[24,25]$ and as coherence decay time in zero magnetic field level crossing (Hanle-effect) experiments, in optical radiofrequency double resonance experiments, and in Zeeman quantum beat experiments [29-31]. $\tau_{0}$ is obtained as coherence decay time from the width of the "broad Hanle signal" ([32] and Sect. 4) and from the width of a microwave optical double resonance signal [33]. Moreover, we obtain $\tau_{0}$ from the width of various "enhanced absorption resonances" ([34-36] and Sect. 5), and from the width of optical absorption lines ([37] and Sect. $6)$. In Sect. 7, we discuss the experimental results and present our conclusion.

\section{Experimental: background and apparatus}

$\mathrm{NO}_{2}$ is a stable molecule having absorption in the visible spectral region $[17,21,22]$. The predominant isotopic form is ${ }^{14} \mathrm{~N}^{16} \mathrm{O}_{2}$. The rotational angular momentum $\mathbf{N}$ couples with the spin $\mathbf{S}$ of the unpaired electron and the nuclear spin $\mathbf{I}$ to the total angular momentum $\mathbf{F}$ following the sequence $\mathbf{N}+\mathbf{S}=\mathbf{J}$ and $\mathbf{J}+\mathbf{I}=\mathbf{F}$. The interaction of the unpaired electron with the rotational angular momentum splits each rotational level into a fine structure doublet with $\mathrm{J}=\mathrm{N} \pm 1 / 2$, and each fine structure level is further split (for $\mathrm{J} \geq 3 / 2$ ) into a triplet with $\mathrm{F}=\mathrm{J}$ and $\mathrm{F}=\mathrm{J} \pm 1$ by nuclear hyperfine interaction. The smallest spacing of levels in this structure is about $50 \mathrm{MHz}$. Fig. 1 is a schematic depiction of two transitions, which we study most. Line 1 is a P-branch transition $(\mathrm{N}+1 \rightarrow \mathrm{N})$ and line $1 \mathrm{R}$ is a R-branch transition $(\mathrm{N}-1 \rightarrow \mathrm{N})$ to the same upper state. Indicated are the angular momentum quantum numbers and gfactors. Fig. 2 of Ref. [27] depicts line 1 and line $1 \mathrm{R}$ as absorption lines in a laser fluorescence excitation spectrum of $\mathrm{NO}_{2}$ near $\lambda_{\text {ex }}=593 \mathrm{~nm}$ under molecular beam conditions. In the experiments, the laser light excites all hfs-levels of a single fine-structure level due to the residual Doppler width in the molecular beam of nearly $100 \mathrm{MHz}$. On the other hand, the $\mathrm{NO}_{2}$ absorption width is $\Delta \nu_{0}=\left(\pi \tau_{0}\right)^{-1}=0.13 \mathrm{MHz}$ (see Sect. 6) which is much smaller than the hfs-splitting in the lower and the upper fine structure level. Therefore, the

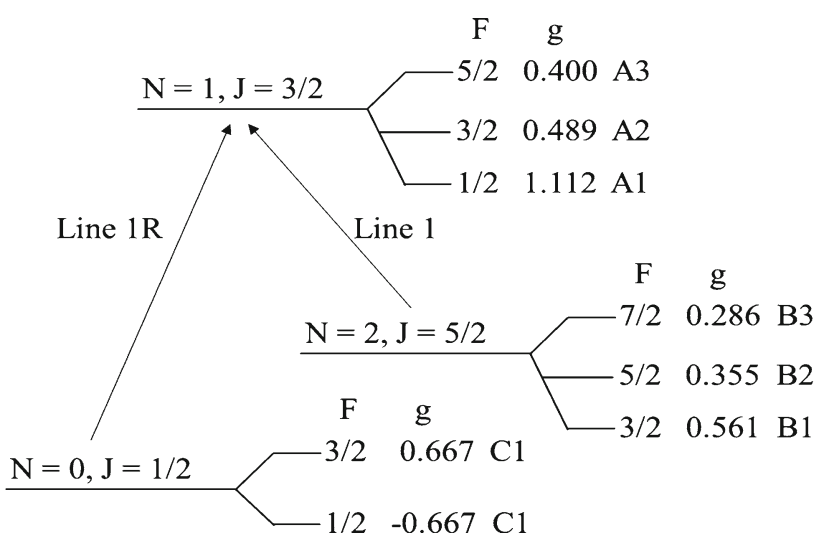

Fig. 1 The laser-induced transitions line $1\left(\lambda^{-1}=\right.$ $\left.16847.76 \mathrm{~cm}^{-1}\right)$ and line $1 \mathrm{R}\left(\lambda^{-1}=16850.29 \mathrm{~cm}^{-1}\right)$ with angular momentum quantum numbers and Landé g-factors (see text). The $A_{i}, B_{i}$, and $C_{i}$ indicate expected resonances for the given g-factors e.g. in Fig. 10. In the literature, the transitions line 1 and line $1 \mathrm{R}$ are often designated $\mathrm{P}(2)$ and $\mathrm{R}(0)$ respectively 
laser light induces transitions between a single hfs-level in the lower state and a single hfs-level in the upper state. The application of the optical rf-double resonance technique (Sect. 3) enables the investigation of a single isolated hfs-level in this molecule. Other experimental techniques, as the Hanle experiment for example, provide in general a superposition of several resonances of which each one is associated with one of the hfs-levels of the excited fine-structure level. On line 1 and line $1 \mathrm{R}$, this superposition comprises only two resonances of which one is by a factor of about 5 larger than the other as the corresponding optical rf-double resonance signals reveal.

Laser light with $\lambda_{\text {ex }} \approx 593 \mathrm{~nm}$ excites $\mathrm{NO}_{2}$ molecules from the electronic ground state $\mathrm{X}^{2} \mathrm{~A}_{1}$ to the excited electronic state $\mathrm{A}^{2} \mathrm{~B}_{2}[21,22]$. At the same excitation energy, there are also other electronic states (B ${ }^{2} \mathrm{~B}_{1}$ and high lying vibrational levels of the electronic ground state $\mathrm{X}^{2} \mathrm{~A}_{1}$ ). The potential energy surfaces (PES) of these electronic states intersect (conical intersection) and enable transitions between these states ([12-16,38,39] and references given there). An example is the intersection of the PES of the electronic states $\mathrm{X}^{2} \mathrm{~A}_{1}$ and $\mathrm{A}^{2} \mathrm{~B}_{2}$ at a particular bond angle that depends on the bond length. At this intersection, the nuclei of the molecule being in the symmetric stretch and bending vibration mode in the $\mathrm{A}^{2} \mathrm{~B}_{2}$ state may pass over into the asymmetric stretch vibration mode in the $\mathrm{X}^{2} \mathrm{~A}_{1}$ state.

An expected eigenstate of the molecule, a hyperfine structure (hfs) level, may be represented by $|\phi\rangle=$ $\mid \mathrm{x}, \mathrm{N}, \mathrm{J}, \mathrm{F}, \mathrm{m}>$, where $\mathrm{m}$ is the quantum number of the projection of $\mathbf{F}$ onto the quantization axis and $\mathrm{x}$ denotes all other quantum numbers, e.g. of the vibrational and electronic structure of the molecule. In an external magnetic field $\mathrm{B}$ directed along the quantization axis, the Zeeman energy of a hfs-level is ([30] and references given there)

$$
\begin{aligned}
& <\phi\left|\mu_{\mathrm{B}} \mathrm{g}_{\mathrm{e}} \mathbf{S} \cdot \mathbf{B}\right| \phi>=\mathrm{g}_{\mathrm{F}} \mu_{\mathrm{B}} \mathrm{mB} \\
& \mathrm{g}_{\mathrm{F}}=\mathrm{g}_{\mathrm{J}} \delta(\mathrm{F}, \mathrm{J}), \mathrm{g}_{\mathrm{J}}= \pm \mathrm{g}_{\mathrm{e}}(2 \mathrm{~N}+1)^{-1} \\
& \delta(\mathrm{F}, \mathrm{J})=\frac{[\mathrm{F}(\mathrm{F}+1)+\mathrm{J}(\mathrm{J}+1)-I(I+1)]}{[2 \mathrm{~F}(\mathrm{~F}+1)]}
\end{aligned}
$$

with $g_{e}=2.002$ the free electron $g$ factor and $\mu_{\mathrm{B}}$ the Bohr magneton. The term $\delta(\mathrm{F}, \mathrm{J})$ results from the coupling $\mathbf{J}+\mathbf{I}=\mathbf{F}$ and the term $\mathrm{g}_{\mathrm{J}}$ results from the coupling $\mathbf{N}+\mathbf{S}=\mathbf{J}$. The + sign is for the fine structure level $\mathrm{J}=\mathrm{N}+1 / 2$ and the - sign for the fine structure level $\mathrm{J}=\mathrm{N}-1 / 2$. In this expression of the Zeeman energy, we neglect terms, which are about three orders of magnitude smaller than the magnetic moment of the electron, e.g. the nuclear magnetic moment and the magnetic moment associated with the molecular rotation. In Ref. [37] (see Fig. 12 of [37]), we measure the g-factors depicted in Fig. 1 simultaneously in the upper and the lower state of the laser-induced transition in agreement (precision 1\%) with the expected values according to Eqs. 2a-2c.
In an optical radiofrequency (rf) double resonance experiment, a rf-field with frequency $\nu_{\text {rf }}$ induces transitions between magnetic sublevels differing by $\Delta \mathrm{m}=$ \pm 1 . A change in the population of the magnetic sublevels of the optically excited state causes a change in the polarization of the fluorescence light. The resonance condition for $\Delta \mathrm{m}= \pm 1$ transitions and the width $\Delta \mathrm{B}$ (HWHM) of this resonance are [40]

$$
\begin{aligned}
& \Delta \mathrm{E}=2 \pi \hbar \nu_{\mathrm{rf}}=\left|\mathrm{g}_{\mathrm{F}}\right| \mu_{\mathrm{B}} \mathrm{B}, \\
& \left|\mathrm{g}_{\mathrm{F}}\right|\left(\mu_{\mathrm{B}} / \hbar\right) \Delta \mathrm{B}=\left\{\left(1 / \tau_{\mathrm{c}}\right)^{2}+\left(\mathrm{w}_{1}\right)^{2}\right\}^{1 / 2}
\end{aligned}
$$

where $\Delta \mathrm{E}$ is the energy spacing of magnetic sublevels differing by $\Delta \mathrm{m}= \pm 1$. The quantity $\mathrm{w}_{1}$ is proportional to the interaction matrix element of the rf field. Extrapolation of $\Delta \mathrm{B}$ to zero rf-field power allows a measurement of the coherence lifetime $\tau_{\mathrm{c}}$. According to Eq. 3a the energy spacing $\Delta \mathrm{E}$ is independent of $\mathrm{m}$. This is valid only if the magnetic field interaction is much smaller than the coupling energy between $\mathbf{J}$ and $\mathbf{I}$, i.e. much smaller than the hfs-splitting of about $50 \mathrm{MHz}$. In a large magnetic field $\mathrm{B}$, the energy spacing $\Delta \mathrm{E}$ of the magnetic sublevels becomes dependent on $\mathrm{m}$, and the width of the magnetic resonance becomes larger than the value $\Delta \mathrm{B}$ in Eq. (3b). Optical-rf double resonance experiments on $\mathrm{NO}_{2}$ with resonance frequencies $\nu_{\mathrm{rf}}>3 \mathrm{MHz}$ reveal already an increase of the width of the resonance signal. At even stronger magnetic fields $\left(\nu_{\mathrm{rf}}>6 \mathrm{MHz}\right)$ there appear several well resolved resonances instead of a single resonance on one hfs- level (for example see Fig. 4 of Ref. [30]).

A very convenient method to investigate the lifetime of coherence $\tau_{c}$ is the Hanle effect or the zero magnetic field level crossing experiment [41]. In this experiment, two or more levels, which cross in energy at zero magnetic field B are coherently excited ( $\sigma$-excitation). The degree of polarization of the fluorescence light shows a resonance as the magnetic field is swept through $\mathrm{B}=0$. The signal has a Lorentzian shape and the width $\Delta \mathrm{B}$ (HWHM) of the resonance depends on $\tau_{\mathrm{c}}$ as follows $[36,41]$

$$
2\left|\mathrm{~g}_{\mathrm{F}}\right|\left(\mu_{\mathrm{B}} / \hbar\right) \Delta \mathrm{B}=1 / \tau_{\mathrm{c}}
$$

The Hanle signal can also be used to measure the sign of the g-factor [30]. In the experiments reported below molecules are excited into a single fine structure level. This has the disadvantage that the Hanle signal is in general a superposition of the three Hanle signals associated with the three hfs-levels of the excited fine structure level.

Figure 2 depicts schematically the two experimental arrangements for the investigations reported in the subsequent sections. We use the one-light-beam setup in Fig. 2a in the experiments in Sects. 3-5 and introduce the coordinate system $\{x, y, z\}$ to describe this setup. A light beam from a cw single-mode dye laser $(\lambda \approx 593 \mathrm{~nm})$ or a $\mathrm{cw}$ single-mode argon ion laser $(\lambda \approx 514 \mathrm{~nm})$ directed along the y-axis excites $\mathrm{NO}_{2}$ molecules propagating in a beam along the $\mathrm{x}$-axis about 
$10 \mathrm{~mm}$ downstream from the nozzle. The crossing of the two beams is the centre of the coordinate system. We detect the laser-induced molecular fluorescence light $\mathrm{F}$ by one or by two photomultipliers in opposite directions along the z-axis. The fluorescence light $\mathrm{F}$ passes a polarizer and a cut-off filter in front of each photomultiplier. We introduce the polarization vectors $\mathbf{e}_{\mathrm{x}}, \mathbf{e}_{\mathrm{y}}$, and $\mathbf{e}_{\mathrm{z}}$ describing linear polarizations along the $\mathrm{x}, \mathrm{y}$, and $\mathrm{z}-$ axis, respectively, and $\mathrm{F}_{\mathrm{x}}$ and $\mathrm{F}_{\mathrm{y}}$ representing the fluorescence light with polarization vectors $\mathbf{e}_{\mathrm{x}}$ and $\mathbf{e}_{\mathrm{y}}$ at the input of the photo detectors. The cut-off filters transmit light with wavelength $\lambda \geq \lambda_{c}$ and suppress light with $\lambda<\lambda_{c}$ by about a factor of $10^{4}$. Thus, the photomultipliers detect fluorescence light (wavelength $\lambda$ ) in the detection window $\lambda_{\mathrm{c}} \leq \lambda \leq \lambda_{0}$, where $\lambda_{0} \approx 850 \mathrm{~nm}$ is set by the sensitivity (S20 cathode) of the photomultipliers. A static magnetic field $\mathrm{B}$ and a rf-field $\mathrm{B}_{1}$ can be applied. The earth magnetic field is compensated. The $B$ field defines the quantization axis and can be directed either along the $\mathrm{z}$ or the $\mathrm{x}$-axis. The $\mathrm{B}_{1}$ field is linearly polarized along the $\mathrm{y}$-axis. The molecular beam is an effusive beam without any collimation. The molecules are leaving a nozzle (diameter $0.1 \mathrm{~mm}$, stagnation pressure $\approx 70$ mbar) into a vacuum with a background pressure of less than $10^{-2} \mu$ bar. As reported in Ref. [29] our first molecular beam apparatus did not enable collisionfree conditions and had to be improved. Most measurements were performed or repeated with the improved apparatus. For instance, the signal, erroneously named "decoupling signal" (see discussion in Ref. [42]), is not seen under the collision-free conditions in the improved molecular beam apparatus.

In the two-light-beams set-up in Fig. 2b, the light beam is split by a $50 \%$ beam splitter into two beams L1 and L2, which both cross the molecular beam. The arrangement of the magnetic fields, the fluorescence detection as well as the molecular beam apparatus are the same as in the set-up discussed before. The angle $\alpha$ can be varied continuously around $\alpha=0$. For $\alpha=0$, both light beams are parallel to each other (at right angle to the molecular beam axis) and are separated by the gap width $\mathrm{s}$. The gap width $\mathrm{s}$ as well as the aperture width $\mathrm{d}$, which determines the diameters of L1 and L2, can be chosen appropriately. For $\alpha=0$, both $\mathrm{s}$ and $\mathrm{d}$ define the time of flight $\left(T_{S}+T_{L}\right)$ of the molecules from the centre of $\mathrm{L} 1$ to the centre of $\mathrm{L} 2$ with $\mathrm{s}=\mathrm{uT}_{\mathrm{s}}$ and $\mathrm{d}=\mathrm{uT}_{\mathrm{L}}$, where $\mathrm{u}=610 \mathrm{~ms}^{-1}$ is the average velocity of the molecules along the molecular beam axis [35]. Here $\mathrm{T}_{\mathrm{L}}$ is the transit time of the molecules through L1 or L2.

The transit time $\mathrm{T}_{\mathrm{L}}$ of the molecules through the light beams L1 or L2 (only L1 in the one-light-beam set-up) is an important parameter in the experiments and requires a reproducible adjustment. In all experiments the light beam passes a beam expander comprising of two lenses (not shown in Fig. 2) before it hits the aperture with width $\mathrm{d}$ and height $\approx 5 \mathrm{~mm}$. We tune the laser light to a chosen absorption line (with $\mathrm{d}$ $>>$ light beam diameter). Varying the distance of the two lenses, L1 (and similar L2) changes from a divergent $(\beta>0)$ to a convergent $(\beta<0)$ light beam at the crossing with the molecular beam. Fig. 3 depicts the
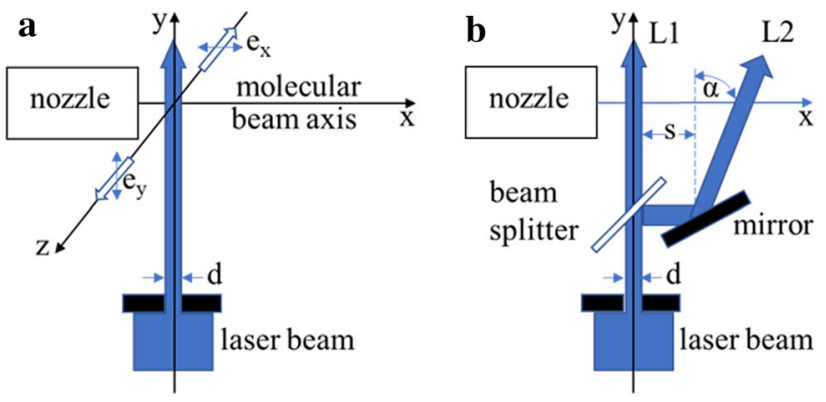

Fig. 2 Schematic diagram of the two experimental arrangements showing in (a) the one-light-beam setup and in (b) the two-light-beams set-up

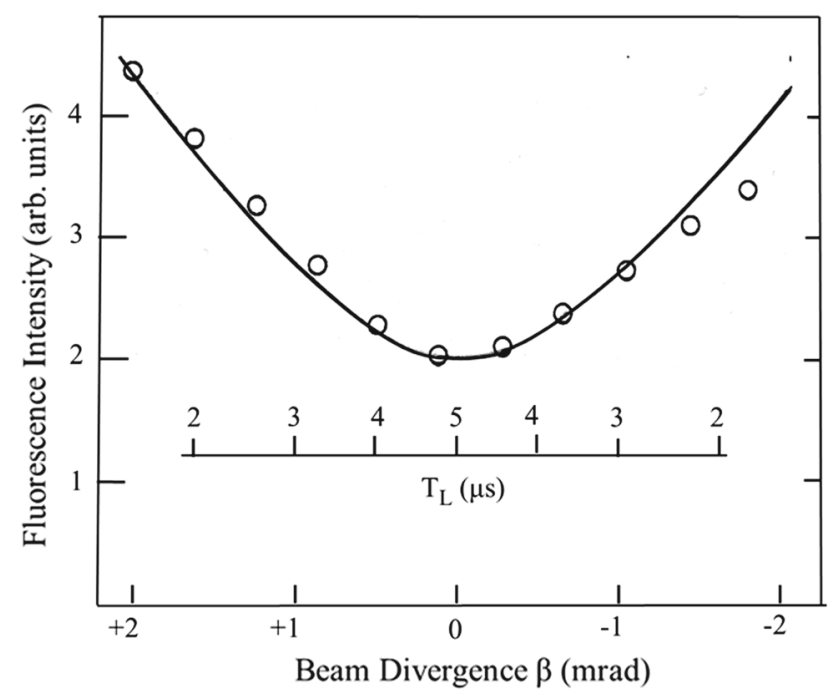

Fig. 3 Fluorescence intensity $F$ versus the divergence (described by the angle $\beta$ ) of the light beam L1 (see text). The solid line is a theoretical curve (see discussion in Sect. 3)

fluorescence intensity $\mathrm{F}$ (no polarizer and one photomultiplier only) versus $\beta$ using only L1 with the laser light tuned to line $1 \mathrm{R}$, and with the light power 500 $\mathrm{mW}$. At the minimum of $\mathrm{F}$, the light beam L1 seems to cross the molecular beam as parallel beam having a rectangular "intersection area" of width $\mathrm{d}=3 \mathrm{~mm}$ and height $\approx 5 \mathrm{~mm}$. As the molecular beam is an effusive beam, the "diameter" of the molecular beam is larger than the "intersection area" of the laser beam at the crossing with the molecular beam also for $\beta>0$. The strong dependence of $\mathrm{F}$ on $\beta$ (up to $50 \%$ ) is characteristic for the experiments on $\mathrm{NO}_{2}$. This dependence is much smaller $(<5 \%)$ in test experiments using $\mathrm{I}_{2}$. The solid line in Fig. 3 is a theoretical curve (see discussion in Sect. 3). We adjust the transit time $\mathrm{T}_{\mathrm{L}}$ using the adjustment of the light beam to a minimum of $\mathrm{F}$, called "adjustment to minimum absorption" in the following. In a first step we minimize $\mathrm{F}$ without the aperture and in a second step we define $T_{L}$ by the aperture width d. 


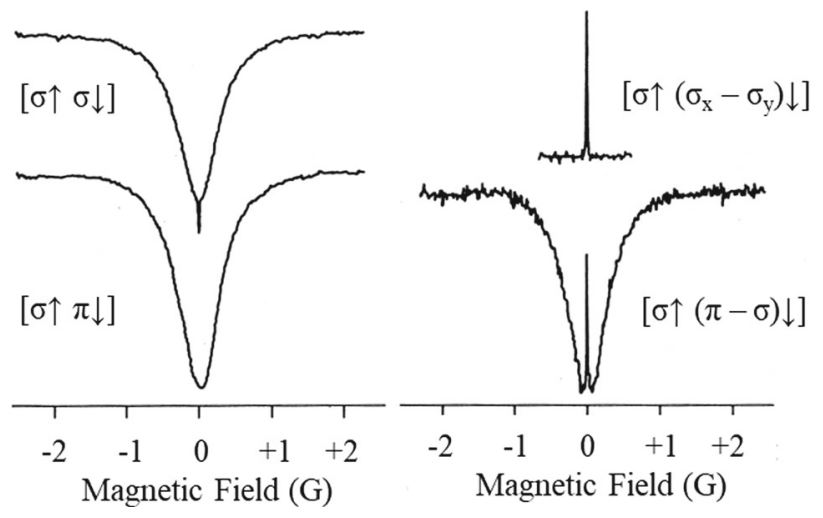

Fig. 4 Magnetic resonance signals in the one-light-beam setup (Fig. 2a) with the laser light tuned to line 1 using four different fluorescence detection arrangements

\section{Inversion effect}

In this section we report on zero magnetic field level crossing (Hanle effect) and optical-radio-frequency double resonance experiments. The most surprising result of these experiments is the inversion effect. We report first on the Hanle experiments. Figure 4 depicts four resonance signals versus the static magnetic field B (no rf-field $\mathrm{B}_{1}$ ) in the one-light-beam setup depicted in Fig. 2a with the laser light tuned to line 1 (see Fig. 1) using four different fluorescence detection arrangements.

a) In the arrangement $[\sigma \uparrow \pi \downarrow]$, the field B is directed along the $\mathrm{x}$-axis. The laser light has linear polarization $\mathbf{e}_{\mathrm{z}}$, representing $\sigma$-polarized excitation (linear polarization perpendicular to the field $\mathrm{B}$ ). Only one photomultiplier is detecting fluorescence light $\mathrm{F}_{\mathrm{x}}$ with linear polarization $\mathbf{e}_{\mathrm{x}}$, which represents $\pi$-polarized fluorescence detection (linear polarization parallel to B).

b) In the arrangement $[\sigma \uparrow \sigma \downarrow l]$, the field $\mathrm{B}$ and the polarization of the laser light are the same as in the arrangement $[\sigma \uparrow \pi \downarrow]$. Only one photomultiplier is detecting fluorescence light $\mathrm{F}_{\mathrm{y}}$ with linear polarization $\mathbf{e}_{\mathrm{y}}$, representing $\sigma$-polarized fluorescence detection.

c) In the arrangement $[\sigma \uparrow(\pi-\sigma) \downarrow]$, the field B and the polarization of the laser light are the same as in arrangement $[\sigma \uparrow \pi \downarrow]$. Fluorescence light $F_{\mathrm{x}}$ and $\mathrm{F}_{\mathrm{y}}$ with linear polarization $\mathbf{e}_{\mathrm{x}}$ and $\mathbf{e}_{\mathrm{y}}$, respectively, is detected with use of both photomultipliers and the difference $F_{x}-F_{y}$ is recorded. The broad resonance signal is about a factor of 10 smaller than the broad resonances in the arrangements $[\sigma \uparrow \sigma \downarrow]$ and $[\sigma \uparrow \pi \downarrow]$, which indicates that $\mathrm{F}_{\mathrm{x}}$ and $\mathrm{F}_{\mathrm{y}}$ differ only little.

d) In the arrangement $\left[\sigma \uparrow\left(\sigma_{\mathrm{x}}-\sigma_{\mathrm{y}}\right) \downarrow\right]$, the field $\mathrm{B} \overline{\text { is }}$ directed along the $\mathrm{z}$ axis. The laser light has linear polarization $\mathbf{e}_{\mathrm{x}}$, representing $\sigma$-polarized excitation. The difference of the fluorescence light $F_{x}-F_{y}$ is recorded. This detection arrangement (called optical bridge [40]) allows to cancel changes of the fluorescence intensity $\mathrm{F}$.
There are no resonance signals with $\pi$-polarized optical excitation. Similar results as in Fig. 4 are obtained on all (about 20) investigated absorption lines near the wavelengths $593 \mathrm{~nm}$ and $514 \mathrm{~nm}[29,34]$. The broad resonance, which we call " $\sigma$-resonance", represents predominantly a change (up to 20\%) of the total fluorescence intensity $\mathrm{F}$ with a minimum of the fluorescence intensity at $\mathrm{B}=0$. The width of the $\sigma$-resonance is associated with the time constant $\tau_{0} \approx 3 \mu \mathrm{s}$ as detailed investigations in Sect. 5 show. In the arrangement $\left[\sigma \uparrow\left(\sigma_{\mathrm{x}}-\sigma_{\mathrm{y}}\right) \downarrow\right]$ we detect solely the narrow resonance which represents a change in the polarization of the fluorescence light as expected for the Hanle signal. The width of the narrow resonance yields a coherence lifetime $\tau_{\mathrm{c}}=\tau_{\mathrm{R}} \approx 35 \mu \mathrm{s}$ as expected for a collisionfree molecule. First Hanle experiments on $\mathrm{NO}_{2}$ used the arrangements $[\sigma \uparrow(\pi-\sigma) \downarrow]$ and $\left[\sigma \uparrow \sigma^{\sigma} \downarrow \downarrow\right]$ and reported lifetimes $\tau_{\mathrm{c}}<<\tau_{\mathrm{R}}$ and no superposition of a narrow and a broad resonance $[43,44]$. For more details see Ref. [34].

In this section we focus on the study of the Hanle signal in the arrangement $\left[\sigma \uparrow\left(\sigma_{\mathrm{x}}-\sigma_{\mathrm{y}}\right) \downarrow\right]$. Figure 5 a depicts measurements of the Hanle signal in this arrangement for three light intensities I with the laser light tuned to line 1 . The measured quantity is

$$
\mathrm{S}=\left(\mathrm{F}_{\mathrm{x}}-\mathrm{F}_{\mathrm{y}}\right) /\left(\mathrm{F}_{\mathrm{x}}+\mathrm{F}_{\mathrm{y}}\right)
$$

versus the magnetic field $\mathrm{B}$. Here $\mathrm{S}$ represents the degree of polarization of the fluorescence light. The Hanle signal in Fig. 5a is a superposition of Hanle signals on the hfs-levels $\mathrm{F}=5 / 2$ and $\mathrm{F}=3 / 2$ of the excited fs-level $(\mathrm{N}=1, \mathrm{~J}=3 / 2)$. The hfs-level $\mathrm{F}=1 / 2$ does not contribute to the Hanle signal because we use excitation with linearly polarized light. The component with $\mathrm{F}=5 / 2$ dominates the Hanle signal (see below). With the g-factor of the $\mathrm{F}=5 / 2$ component solely we obtain $\tau_{\mathrm{c}}=\tau_{\mathrm{R}} \approx 35 \mu \mathrm{s}$ [29]. The unexpected result is the change of the Hanle signal from an upward-directed to a downward-directed signal versus the light intensity at constant line shape and width. We call this change of the signal "inversion effect" [27].

Figure 5b depicts results of an optical-rf double resonance experiment in the one-light-beam setup depicted in Fig. 2a with the laser light tuned to line 1. The magnetic field $\mathrm{B}$ is directed along the $\mathrm{x}$-axis and the rf- field $\mathrm{B}_{1}$ (frequency $\nu_{\mathrm{rf}}=1.4 \mathrm{MHz}$ ) is linearly polarized along the $\mathrm{y}$-axis. The laser light has linear polarization $\mathbf{e}_{\mathrm{x}}$ representing $\pi$-polarized excitation. Figure $5 \mathrm{~b}$ represents the signal S given in Eq. 5 versus the magnetic field B. The measured g-factors agree well with the expected values (Eq. 2a,b) of the upper state hfs-levels $\mathrm{F}=5 / 2$ (the stronger resonance) and $\mathrm{F}=3 / 2$. There is no resonance associated with the hfs-level $\mathrm{F}=1 / 2$ because we use optical excitation with linearly polarized light. The resonance on the hfs-level $\mathrm{F}=5 / 2$ clearly shows the inversion effect versus the light intensity. The width of this signal yields a coherence lifetime $\tau_{\mathrm{c}}=\tau_{\mathrm{R}}$ which agrees well with the result of the Hanle experiment. The results in Fig. 5b are obtained using low power of the rf-field. At high power of the rf-field there is 

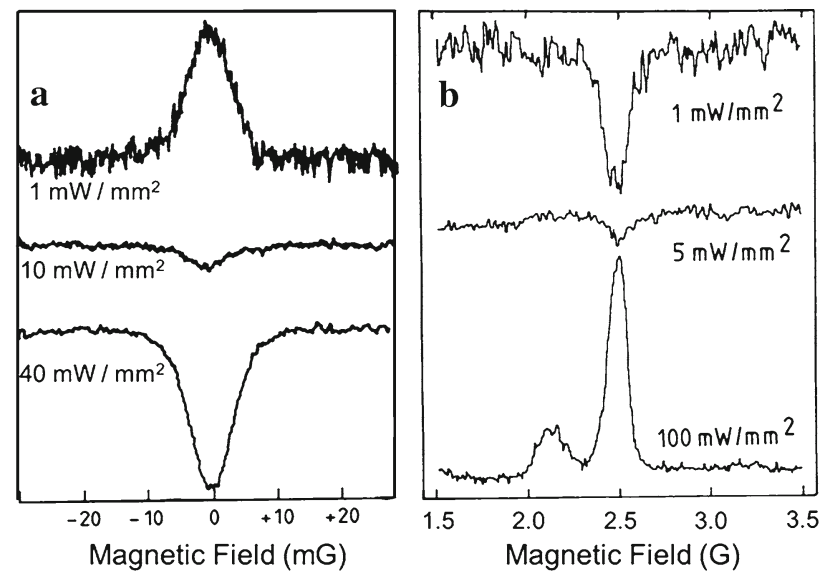

Fig. 5 The Hanle signal (Fig. 5a) and the optical-rf double resonance signal (Fig. 5b) for three light intensities I on line 1 in the one-light-beam setup revealing the inversion effect

additionally to the optical-rf double resonance signals also a broad resonance structure, which is a superposition of several broad resonances (see Fig. 2 in Ref. $[45])$. We discuss these broad resonances in Sect. 5 and show that the width of these resonances (called "broad rf-resonances") is associated with the time constant $\tau_{0} \approx 3 \mu \mathrm{s}$.

An inversion effect does not appear on all absorption lines of $\mathrm{NO}_{2}$ [27]. Figure 6 depicts the signal amplitude versus the light intensity on line 1 and line $1 \mathrm{R}$ in the optical-rf double resonance experiment in the upper state $\mid \mathrm{N}=1, \mathrm{~J}=3 / 2, \mathrm{~F}=5 / 2>$ (see Fig. 1). An inversion effect appears on line 1 but not on line 1R. Hanle experiments on line 1 and line $1 \mathrm{R}$ reveal the same results. The solid lines in Fig. 6 are theoretical curves which we discuss in the following. We describe the experimental results in Figs. 5 and 6 using the model of the space-coupled molecule introduced in Sect. 1 (Eqs. $1 \mathrm{a}-1 \mathrm{c})$. The optical transition $|\mathrm{g}, 1>\leftrightarrow| \mathrm{e}, 1>$ prepares a molecule in $|\mathrm{e}, 1\rangle$, but the molecule evolves in the non-radiative transition $|\mathrm{e}, 1>\rightarrow| \mathrm{e}, 2>$ to the state $\mid \mathrm{e}, 2>$. The fluorescence decay of $\mid \mathrm{e}, 2>$ is detected. The transition $|\mathrm{e}, 1>\rightarrow| \mathrm{e}, 2>$ is associated with the time constant $\tau_{0} \approx 3 \mu$ s which is short compared to the radiative lifetime $\tau_{\mathrm{R}} \approx 35 \mu \mathrm{s}$ of both $\mid \mathrm{e}, 1>$ and $\mid \mathrm{e}, 2>$. In Sect. 4 we discuss the assumption that $\mid \mathrm{e}, 1>$ and $\mid \mathrm{e}, 2>$ have about the same radiative lifetime $\tau_{\mathrm{R}}$, and we study the fluorescence decay of $\mid e, 1>$. To simplify the writing of the following equations, we introduce

$$
\begin{aligned}
& |\mathrm{g}, 1, \mathrm{~m}>=| \mathrm{a}, \mathrm{m}>,|\mathrm{e}, 1, \mathrm{~m}>=| \mathrm{b}, \mathrm{m}> \\
& |\mathrm{e}, 2, \mathrm{~m}>=| \mathrm{c}, \mathrm{m}>
\end{aligned}
$$

with $\mathrm{m}$ labelling the magnetic sublevels and with $\mathrm{a}_{\mathrm{m}}$, $b_{m}$, and $c_{m}$ representing the occupation probabilities of $|\mathrm{g}, 1, \mathrm{~m}>,| \mathrm{e}, 1, \mathrm{~m}>$, and $\mid \mathrm{e}, 2, \mathrm{~m}>$, respectively. Furthermore, we assume an optical transition with $\Delta \mathrm{m}=0$ as in the optical-rf double resonance experiment and propose the following rate equations for the occupation

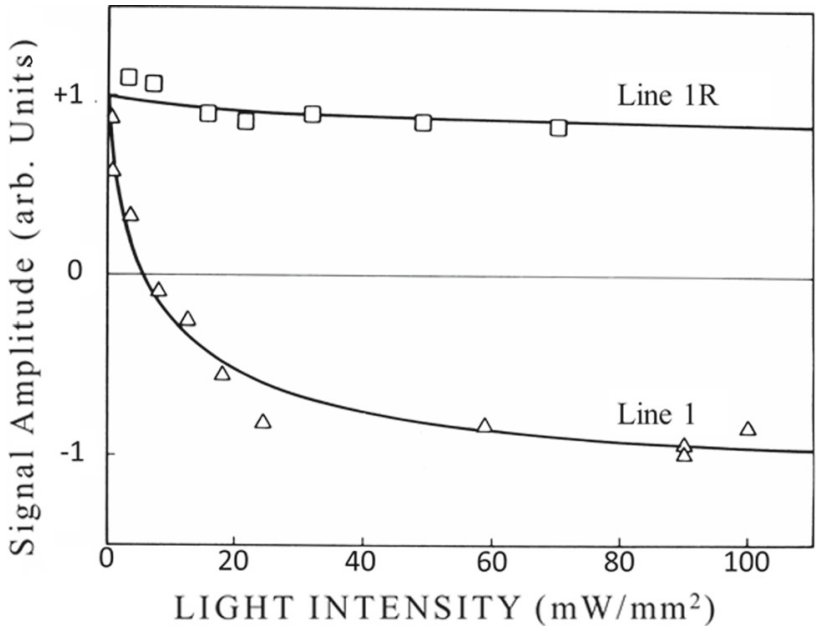

Fig. 6 The signal amplitude versus the light intensity on line 1 and line $1 \mathrm{R}$ in the optical-rf double resonance experiment in the upper state $\mid \mathrm{N}=1, \mathrm{~J}=3 / 2, \mathrm{~F}=5 / 2>$ (see Fig. 1)

probabilities:

$$
\begin{aligned}
\dot{a}_{m}= & \left(1 / T_{L}\right)\left(\rho^{0}-a_{m}\right)-\gamma_{m}\left(a_{m}-b_{m}\right) \\
\dot{b}_{m}= & \gamma_{m}\left(a_{m}-b_{m}\right)-\left[\lambda_{m}+\left(1 / \tau_{R}\right)\right] b_{m} \\
\dot{c}_{m}= & \lambda_{m} b_{m}-\left(1 / \tau_{R}\right) c_{m} \\
\lambda_{m}= & \left(1 / \tau_{0}\right)\left(1+R \tau_{0} \gamma_{m}\right)^{-1} \\
& R=\left[1-\exp \left(-T_{L} / \tau_{0}\right)\right]
\end{aligned}
$$

Here $\rho^{0}$ represents the population of $|\mathrm{g}, 1, \mathrm{~m}\rangle=\mid \mathrm{a}, \mathrm{m}>$ before an interaction with the light beam. $\mathrm{T}_{\mathrm{L}}$ is the transit time of the molecules through the light beam. $\gamma_{\mathrm{m}}$ is the rate of the laser induced transition $|\mathrm{g}, 1, \mathrm{~m}>\leftrightarrow| \mathrm{e}, 1, \mathrm{~m}>$, and $\lambda_{\mathrm{m}}$ is the rate of the transition $|\mathrm{e}, 1, \mathrm{~m}>\rightarrow| \mathrm{e}, 2, \mathrm{~m}>$. We assume $\lambda_{\mathrm{m}}>\left(1 / \tau_{\mathrm{R}}\right)$ and neglect the radiative decay of $\mid e, 1>$. The above rate equations are introduced in Ref.[27] with the quantity $\mathrm{R} \tau_{0}$ in $\lambda_{\mathrm{m}}$ as unknown constant, which is fitted to the experimental results. R given by Eq. $7 \mathrm{~d}$ is derived in later work (Ref.[46], see Appendix A).

The results in Fig. 6 differ for line 1 (P-branch transition, $\mathrm{F}+1 \rightarrow \mathrm{F}$ ) and line $1 \mathrm{R}$ (R-branch transition, $\mathrm{F}-1 \rightarrow \mathrm{F}$ ) because the transition rates $\gamma_{\mathrm{m}}$ to the upper state $\mid \mathrm{N}=1, \mathrm{~J}=3 / 2, \mathrm{~F}=5 / 2>$ differ. Following Ref. [27], we write $\gamma_{\mathrm{m}}=\gamma_{\mathrm{m}, \alpha}$ with $\alpha=\mathrm{P}, \mathrm{R}$ indicating the $\mathrm{P}$ - or R-branch transition and factorize both into $\gamma_{\mathrm{m}, \alpha}=\mathrm{x}_{\mathrm{m}, \alpha} \gamma_{\alpha}$ with $\gamma_{\alpha}$ being independent of the quantum number $\mathrm{m}$, and with $\mathrm{x}_{\mathrm{m}, \mathrm{R}}=\left\{\mathrm{F}^{2}-\mathrm{m}^{2}\right\}$ and $\mathrm{x}_{\mathrm{m}, \mathrm{P}}=$ $\left\{(\mathrm{F}+1)^{2}-\mathrm{m}^{2}\right\}$. Under stationary state conditions we obtain from Eqs. $(7 \mathrm{a})-(7 \mathrm{~d})$ with $\tau=\left(\tau_{0}+\mathrm{T}_{\mathrm{L}}\right)$ :

$$
c_{\mathrm{m}}=\frac{\left(\rho^{0} \tau_{\mathrm{R}} / \tau_{0}\right)\left(\mathrm{x}_{\mathrm{m}, \alpha} \gamma_{\alpha} \tau_{0}\right)}{1+\left(\mathrm{x}_{\mathrm{m}, \alpha} \gamma_{\alpha} \tau\right)+\mathrm{R}\left(\mathrm{x}_{\mathrm{m}, \alpha} \gamma_{\alpha} \tau_{0}\right)^{2}}
$$

With these occupation probabilities, we calculate the signal amplitude $S(0)-S(\infty)$ on line 1 and line $1 R$ 
with $\mathrm{S}$ given by Eq. 5 . Here $\mathrm{S}(0)$ means $\mathrm{S}$ at resonance and $S(\infty)$ means $S$ far off-resonance. In the Hanle signal, we have $\mathrm{S}(0)$ at $\mathrm{B}=0$ and $\mathrm{S}(\infty)$ at $\mathrm{B}>>0$. The strong magnetic field perturbs the Zeeman coherence and causes the fluorescence light being independent of a rotation about the direction of the magnetic field $\mathrm{B}$, the z-axis. This gives $\mathrm{F}_{\mathrm{x}}=\mathrm{F}_{\mathrm{y}}$ and consequently $\mathrm{S}(\infty)=0$. Therefore, in the Hanle experiment, $\mathrm{S}(0)$ determines solely the signal amplitude and represents the degree of polarization of the fluorescence light unperturbed by the magnetic field B. On the other hand, in the opticalrf double resonance experiment, we have $\mathrm{S}(0)$ if the Zeeman splitting of the magnetic sublevels is in resonance with the rf-field. The rf-transitions cause equal occupation probabilities in all magnetic sublevels, which gives $\mathrm{F}_{\mathrm{x}}=\mathrm{F}_{\mathrm{y}}$ and $\mathrm{S}(0)=0$ at resonance. Far off-resonance, we can neglect the rf-field and $\mathrm{S}(\infty)$ represents the degree of polarization of the fluorescence light. Therefore, contrary to the Hanle experiment, $S(\infty)$ determines the signal amplitude in the optical-rf double resonance experiment. This explains the different "signs" of the amplitudes of both signals at low light intensity. $\mathrm{S}(0)$ in the Hanle experiment and $\mathrm{S}(\infty)$ in the optical-rf double-resonance experiment (with $\pi$-excitation) differ only in the "sign". In Ref. [27], we calculate $S(0)$ in the upper hfs-level $\mid \mathrm{N}=1, \mathrm{~J}=3 / 2, \mathrm{~F}=5 / 2>$ for excitation with line 1 and line $1 \mathrm{R}$

$$
S(0)=2 \mathrm{w} \frac{4 c_{1 / 2}+c_{3 / 2}-5 c_{5 / 2}}{c_{1 / 2}+c_{3 / 2}+c_{5 / 2}}
$$

with the $c_{m}$ given in Eq. 8 and with $c_{m}=c_{-m}$. The quantization axis is along the polarization direction of the exciting light beam. The quantity w can be written as a sum of three terms with $\mathrm{w}=\mathrm{w}_{\mathrm{P}}-\mathrm{w}_{\mathrm{Q}}+\mathrm{w}_{\mathrm{R}}$, where the subscripts $\mathrm{P}, \mathrm{Q}$, and $\mathrm{R}$ stand for $\mathrm{P}-, \mathrm{Q}-$, and R-branch transitions in the fluorescence decay of $|\mathrm{e}, 2>=| \mathrm{c}>$. The quantity $\mathrm{w}$ is independent of the excitation process and is therefore not of direct interest for the present discussion. We come back to the quantity w in Sect. 4. The two solid lines in Fig. 6 represent a fit of $\mathrm{S}(0)$ given by Eq. 9 to the experimental results. On line $1 \mathrm{R}$, we have $c_{5 / 2}=0$ because $\mathrm{x}_{\mathrm{m}, \mathrm{R}}=\left\{\mathrm{F}^{2}-\mathrm{m}^{2}\right\}=0$ for $\mathrm{m}=5 / 2$. Therefore, $\mathrm{S}(0)$ does not change the sign on line 1R. The details of this work are described in Ref. [27], where we introduce $\left\{\mathrm{R}\left(\tau_{0} / \tau\right)^{2}\right\}=\mathrm{A}$ and $\left\{\tau \gamma_{\alpha}\right\}=D_{\alpha} \mathrm{I}$ with I denoting the light intensity. In Ref. [27], we adjust $\mathrm{D}_{\alpha}$ and $\mathrm{A}$ to fit $\mathrm{S}(0)$ to the experimental results. The fit gives $\mathrm{A}=0.1$. Here, we estimate the value $\mathrm{A}=\left\{\left[1-\exp \left(-\mathrm{T}_{\mathrm{L}} / \tau_{0}\right)\right]\left(\tau_{0} / \tau\right)^{2}\right\}$. The light beam crossing the molecular beam has a diameter $\mathrm{d}=1.5 \mathrm{~mm}$ which gives $\mathrm{T}_{\mathrm{L}}=\mathrm{d} / \mathrm{u}=2.4 \mu \mathrm{s}$. With $\tau_{0}=3 \mu \mathrm{s}$ we obtain $\tau=\left(\tau_{0}+\mathrm{T}_{\mathrm{L}}\right)=5.4 \mu \mathrm{s},\left(\tau_{0} / \tau\right)^{2}=0.3$, and finally $\mathrm{A}=0.16$, which agrees quite well with the fit in Ref. [27]. We note that the "adjustment to minimum absorption" of the light beam (see Sect. 2 and Fig. 3 ) is not used in the measurements in Figs. 5 and 6, because this adjustment is introduced in later work [35]. However, the light beam in the measurements in Figs. 5 and 6 is not focused onto the molecular beam but crosses this beam approximately as parallel light beam. There is an inversion effect also at constant light intensity I versus the transit time $T_{L}$ of the molecules through the light field of the laser (see Fig. 8 in Sect. 4).

In Eqs. 7-9, we calculate the inversion effect based on the "allowed" optical transitions with $\Delta \mathrm{N}=\Delta \mathrm{J}=$ $\Delta \mathrm{F}= \pm 1$ for parallel bands in the ${ }^{2} \mathrm{~B}_{2} \leftarrow{ }^{2} \mathrm{~A}_{1}$ excitation. However, as pointed out in Ref. [28], "forbidden" transitions with $\Delta \mathrm{F}=0$ are not unusual and are also measured in $\mathrm{NO}_{2}$ additionally to the strong "allowed" transitions, and an overlap of $\Delta \mathrm{F}= \pm 1$ and $\Delta \mathrm{F}=0$ optical transitions may eventually affect the inversion effect. Ref. [28] analyses a Lamb dip spectrum with high resolution taken on an optical transition (line 3 in Ref.[27]) which reveals the inversion effect on all three hfs-levels. This spectrum allows to identify all possible optical transitions between single hyperfine structure levels in the lower and the upper state. Ref. [28] concludes that there is at least one hfs-level which reveals the inversion effect and is excited by a single $\Delta \mathrm{F}=-1$ transition solely. This proves that the inversion effect does not depend on an overlap of $\Delta \mathrm{F}= \pm 1$ and $\Delta \mathrm{F}=0$ transitions. We do not exclude the presence of "forbidden" optical transitions, but these transitions are not the cause of the inversion effect as Ref. [28] shows.

There is no inversion effect for $\mathrm{R}=0$ in Eq. $7 \mathrm{~d}$, i.e. for $T_{L}<<\tau_{0}$. The quantity $R$ is responsible also for the strong dependence of the fluorescence intensity $\mathrm{F}$ on the divergence of the light beam in Fig. 3. The measurements depicted in Fig. 3 use line $1 \mathrm{R}$ and the light power $500 \mathrm{~mW}$. At the minimum of the fluorescence intensity $\mathrm{F}$, the light beam crosses the molecular beam as parallel beam with the diameter $\mathrm{d}=3 \mathrm{~mm}$ which corresponds to a transit time $\mathrm{T}_{\mathrm{L}}=\mathrm{d} / \mathrm{u}=5 \mu \mathrm{s}$ with $\mathrm{u}=610 \mathrm{~ms}^{-1}$. F is proportional to $\Sigma_{\mathrm{m}} \mathrm{c}_{\mathrm{m}}$ if we neglect the radiative decay of $\mid e, 1>$. Due to the high light power, we expect $\left(\mathrm{x}_{\mathrm{m}, \alpha} \gamma_{\alpha} \tau_{0}\right)>>1$ and therefore $\mathrm{c}_{\mathrm{m}} \approx\left(\rho^{0} \tau_{\mathrm{R}} / \tau_{0}\right)\left\{\left(\mathrm{x}_{\mathrm{m}, \alpha} \gamma_{\alpha} \tau_{0}\right) \mathrm{R}\right\}^{-1}$ in Eq. 8. This gives $\mathrm{F}=\mathrm{F}_{0} / \mathrm{R}$, where $\mathrm{F}_{0}$ is constant in the measurements in Fig. 3. The solid line in Fig. 3 represents the quantity $\mathrm{F}=\mathrm{F}_{0} / \mathrm{R}$ versus $\mathrm{T}_{\mathrm{L}}$ (see Eq. $7 \mathrm{~d}$ ) with $\mathrm{F}_{0}$ fitted to the experimental results at the minimum of $\mathrm{F}$ and with $\mathrm{T}_{\mathrm{L}}=5 \mu \mathrm{s}$. Figure 3 shows that a beam divergence $\beta \neq 0$ causes an effective shortening of the transit time $\mathrm{T}_{\mathrm{L}}$.

The rate constant $\lambda_{\mathrm{m}}$ with the quantity $\mathrm{R}$ in Eq. $7 \mathrm{~d}$ is calculated for molecules travelling through the light beam in the time $T_{L}=d / u$ along a plane wavefront (a surface of constant phase) [46]. Obviously, this condition is approximately realized if the divergence of the light beam is adjusted to the minimum of the fluorescence intensity $\mathrm{F}$ (adjustment to minimum absorption) in Fig. 3. At this minimum of $\mathrm{F}$, the light-induced optical coherence in the optical transition $|\mathrm{g}, 1>\leftrightarrow| \mathrm{e}, 1>$ seems to be in phase with the optical field for a maximum time enabling many cycles of absorption and induced emission in the transition $|\mathrm{g}, 1>\leftrightarrow| \mathrm{e}, 1>$. This reduces the effective light absorption in the molecular beam and explains the results in Fig. 3. The same mechanism also explains the inversion effect. For small values 
of light intensity I and transit time $\mathrm{T}_{\mathrm{L}}$, the probability for induced emission is too small to reduce $\lambda_{\mathrm{m}}$ and we have $\lambda_{\mathrm{m}} \approx 1 / \tau_{0}$, i.e. the transition $|\mathrm{e}, 1>\rightarrow| \mathrm{e}, 2>$ is independent of the quantum number $\mathrm{m}$, and the states $\mid \mathrm{e}, 1>$ and $\mid \mathrm{e}, 2>$ have the same occupation probability distribution in the magnetic sublevels. Those magnetic sublevels $\mid \mathrm{e}, 1, \mathrm{~m}>$ and $\mid \mathrm{e}, 2, \mathrm{~m}>$ have maximum occupation probability, for which the laser induced transition rate $\gamma_{\mathrm{m}}$ is maximum. However, for large values of $\mathrm{I}$ and $\mathrm{T}_{\mathrm{L}}$, the transition rate $\lambda_{\mathrm{m}}$ depends on $\mathrm{m}$ and is approximately proportional to $\left(1 / \gamma_{\mathrm{m}}\right)$. The larger $\gamma_{\mathrm{m}}$, the less is the population of $|\mathrm{e}, 2, \mathrm{~m}\rangle$. This causes the inverse population distribution in the $\mid \mathrm{e}, 2, \mathrm{~m}>$ for high values of $I$ and $T_{L}$. The inversion effect reveals that the process driving the transition $|\mathrm{e}, 1>\rightarrow| \mathrm{e}, 2>$ does not affect the coherence in the optical transition |g, $1>\leftrightarrow \mid \mathrm{e}, 1>$.

\section{The broad Hanle signal}

The Hanle signal and the optical-rf double resonance signal discussed in Sect. 3 have both a width characteristic of the radiative lifetime $\tau_{\mathrm{R}} \approx 35 \mu$ s of the excited state. We associate both signals with the state $\mid \mathrm{e}, 2>$. In this section we report on a Hanle signal which we assign to the state $|\mathrm{e}, 1\rangle$. This signal is called the broad Hanle signal because its width is by the factor $\tau_{R} / \tau_{0} \approx 10$ broader than the width of the Hanle signal discussed in Sect. 3 which we call the narrow Hanle signal in the following.

As described in Sect. 2, we detect the laser induced molecular fluorescence (wavelength $\lambda$ ) in the detection window $\lambda_{\mathrm{c}} \leq \lambda \leq \lambda_{0}$ with $\lambda_{\mathrm{c}}$ given by cut-off filters and with $\lambda_{0} \approx 850 \mathrm{~nm}$ given by the sensitivity of the photomultipliers. We use $\lambda_{c}=630 \mathrm{~nm}$ in the Hanle and optical-rf double resonance experiments discussed in Sect. 3. In the following, we report on Hanle experiments with $\lambda_{\mathrm{c}}=630 \mathrm{~nm}, 715 \mathrm{~nm}$, and $780 \mathrm{~nm}$ [32]. Figure 7 depicts results of a Hanle experiment on line 1 using the same experimental set up (arrangement $\left.\left[\sigma \uparrow\left(\sigma_{\mathrm{x}}-\sigma_{\mathrm{y}}\right) \downarrow\right]\right)$ as the Hanle experiment in Fig. 5a except two changes, namely $\lambda_{c}=715 \mathrm{~nm}$ instead of $\lambda_{\mathrm{c}}=630 \mathrm{~nm}$ and $\mathrm{T}_{\mathrm{L}}=\mathrm{d} / \mathrm{u}=8.2 \mu \mathrm{s}$ instead of $\mathrm{T}_{\mathrm{L}}=2.4 \mu \mathrm{s}$. The dots superimposed on both measurements in Fig. 7 represent the sum of two Lorentzian curves, which are fitted to the measurements. The narrow Lorentzian curve has the width (HWHM) of 4 $\mathrm{mG}$, which correspond to a coherence decay time $\tau_{\mathrm{c}}=$ $35 \pm 5 \mu \mathrm{s}$, and the broad Lorentzian curve has the width of $46 \mathrm{mG}$, which corresponds to $\tau_{\mathrm{c}}=2.8 \pm 1.0 \mu \mathrm{s}$ if we assume the same relation between coherence decay time and width for both components (see Eq. 4). The narrow signal shows the inversion effect but not the broad signal. Both signals represent a change of the polarization and not of the intensity of the fluorescence light. Both signals appear as dispersion shaped signals if the polarizers in the fluorescence detection path are tilted by $\pi / 4$ about the z-axis. We perform the same measurements on line $1 \mathrm{R}$ and obtain again a superposition

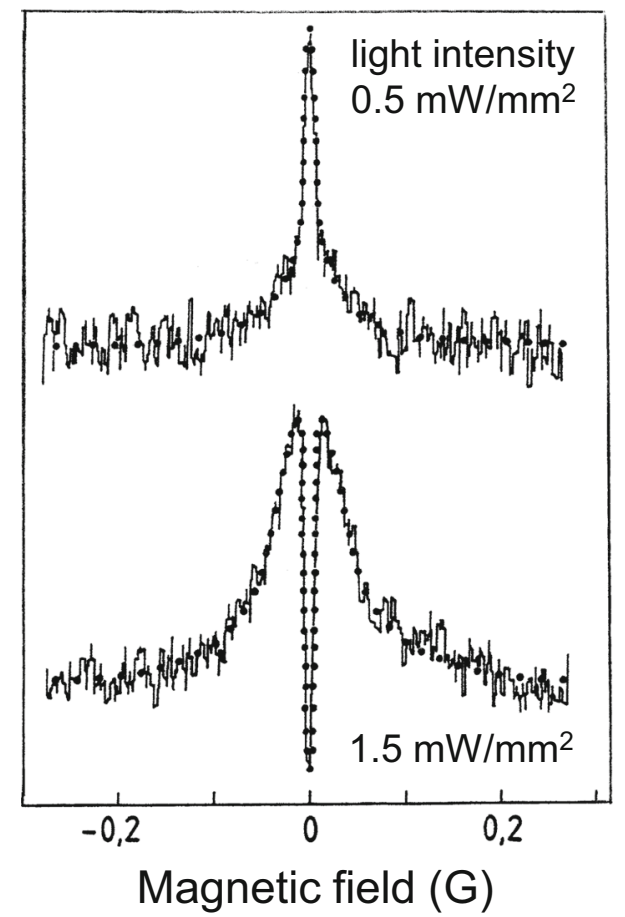

Fig. 7 The Hanle signal on line 1 using experimental conditions different from Fig. 5A (see text). The dots superimposed on the measurements are the sum of two Lorentzian curves

of two Lorentzian shaped resonances having the same widths as on line 1 , however, with no inversion effect of the narrow resonance signal. We identify the narrow Lorentzian curve with the Hanle signal studied in Sect. 3 (now called narrow Hanle signal) and associate it with the state $\mid \mathrm{e}, 2>$ and we call the broad Lorentzian curve the broad Hanle signal and associate it with the state $\mid \mathrm{e}, 1>$. Moreover, we identify the coherence decay time $\tau_{\mathrm{c}} \approx 3 \mu \mathrm{s}$ of the broad Hanle signal with the time constant $\tau_{0}$ of the transition $|\mathrm{e}, 1>\rightarrow| \mathrm{e}, 2>$.

Figure 8 depicts measurements of the amplitude $\mathrm{S}_{0}$ of the signal $\mathrm{S}$ (see Eq. 5) for the narrow and the broad Hanle signal, and of the width $\Delta \mathrm{B}$ (HWHM) of the broad Hanle signal versus the aperture width d with the laser light tuned to line 1 , with the light intensity $\mathrm{I}=1 \mathrm{~mW} / \mathrm{mm}^{2}$, and with $\lambda_{\mathrm{c}}=715 \mathrm{~nm}$. The two amplitudes are not in scale. At $\mathrm{d}=15 \mathrm{~mm}$, corresponding to a transit time $\mathrm{T}_{\mathrm{L}}=\mathrm{d} / \mathrm{u}=25 \mu \mathrm{s}$, the absolute value for $\mathrm{S}_{0}$ is about $5 \cdot 10^{-3}$ for the narrow Hanle signal and $2 \cdot 10^{-3}$ for the broad Hanle signal. In a similar experiment we measure the same quantities versus the light intensity I between $\mathrm{I}=2 \mathrm{~mW} / \mathrm{mm}^{2}$ and $\mathrm{I}=10 \mathrm{~mW} / \mathrm{mm}^{2}$ with $\lambda_{\mathrm{c}}=715 \mathrm{~nm}$ and $\mathrm{d}=5 \mathrm{~mm}$. Both measurements show that the signal amplitude of the broad Hanle signal increases proportional to $\mathrm{d}$ and I, whereas the signal amplitude of the narrow Hanle signal shows the inversion effect versus $d$ and I. The widths of both signals are independent of $d$ and I. We note that the measurement of the broad Hanle signal with $\mathrm{d}=3 \mathrm{~mm}$ needs a data acquisition time of one 


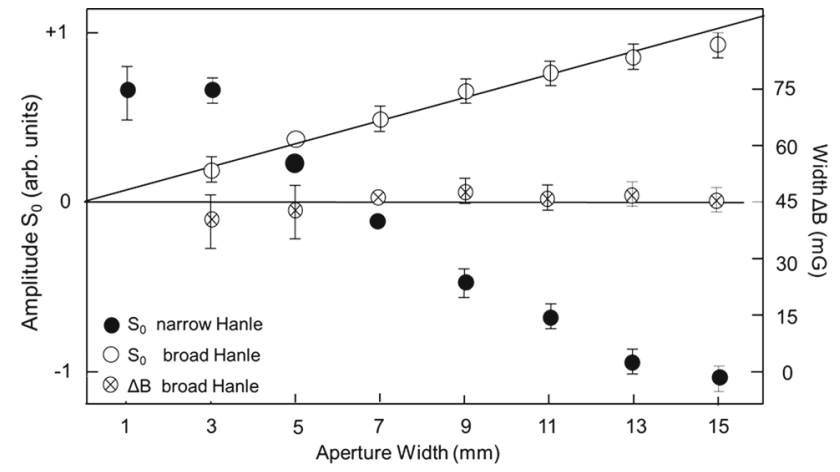

Fig. 8 The amplitude $S_{0}$ of the narrow and the broad Hanle signal, and the width $\Delta \mathrm{B}$ (HWHM) of the broad Hanle signal versus the aperture width $d$ on line 1

hour. A measurement of the broad Hanle signal with an aperture width less than $\mathrm{d}=3 \mathrm{~mm}$ was not feasible.

The superposition of a narrow and a broad Hanle signal is measured on all (10) investigated absorption lines. Detailed measurements as on line 1 and line $1 \mathrm{R}$ are also performed on line 4 and line 8 in the excitation spectrum shown in Fig. 2 of Ref. [30]. The widths of the broad Hanle signals reveal the coherence decay times $\tau_{\mathrm{c}}=2.8 \mu \mathrm{s}$ (line 1 and line $1 \mathrm{R}$ ), $\tau_{\mathrm{c}}=1.7 \mu \mathrm{s}$ (line 4 ), and $\tau_{\mathrm{c}}=2.5 \mu_{\mathrm{s}}$ (line 8) (see Table 1 of Ref. [42]). However, the evaluation of the coherence decay time from the width is strongly hampered on lines 4 and 8 , because all three hfs-levels of these absorption lines contribute to the Hanle signal with g-factors which differ up to a factor 1.6 as optical-rf double resonance measurements on the lines 4 and 8 show [30]. We estimate an uncertainty of $50 \%$ for the coherence decay times on lines 4 and 8 , larger than the uncertainty indicated in Ref.[42]. This is different on lines 1 and $1 \mathrm{R}$ because essentially only one hfs-level of these absorption lines contributes to the Hanle signal (see Sect. 3).

Figure 9 depicts the signal amplitude of the narrow $\left(\mathrm{S}_{\mathrm{n}}\right)$ and the broad $\left(\mathrm{S}_{\mathrm{b}}\right)$ Hanle signal measured in the three detection windows with $\lambda_{\mathrm{c}}=630 \mathrm{~nm}, 715 \mathrm{~nm}$, and $780 \mathrm{~nm}$, and with both amplitudes normalized to the total fluorescence intensity for the given $\lambda_{c}$. In these measurements, the laser light is tuned to line 1 , the light intensity is $\mathrm{I}=1 \mathrm{~mW} / \mathrm{mm}^{2}$, and the transit time is $T_{L}=18 \mu \mathrm{s}(\mathrm{d}=11 \mathrm{~mm})$. The experiments show that $\mathrm{S}_{\mathrm{b}}$ is independent of $\lambda_{\mathrm{c}}$, whereas $\mathrm{S}_{\mathrm{n}}$ decreases strongly versus $\lambda_{c}$. Moreover, the widths of both Hanle signals are independent of $\lambda_{c}$. We note that the broad Hanle signal was not seen in the previous Hanle experiments with $\lambda_{\mathrm{c}}=630 \mathrm{~nm}$ and $\mathrm{d}=1.5 \mathrm{~mm}$ (e.g. Fig. 5a), because $S_{b} / S_{n}<0.1$ and because the magnetic field scan was chosen too narrow for the much "broader" broad Hanle signal.

If we assume that $\mid \mathrm{e}, 1>$ and $\mid \mathrm{e}, 2>$ have about the same radiative lifetime $\tau_{R}$ and the same degree of polarization of the fluorescence light, we may expect $\mathrm{S}_{\mathrm{b}} / \mathrm{S}_{\mathrm{n}} \approx \tau_{0} / \tau_{\mathrm{R}} \approx 0.1$, which agrees about with the result at $\lambda_{c}=630 \mathrm{~nm}$ in Fig. 9. However, the ratio $\mathrm{S}_{\mathrm{b}} / \mathrm{S}_{\mathrm{n}}$ increases with $\lambda_{\mathrm{c}}=715 \mathrm{~nm}$ and $\lambda_{\mathrm{c}}=780 \mathrm{~nm}$,

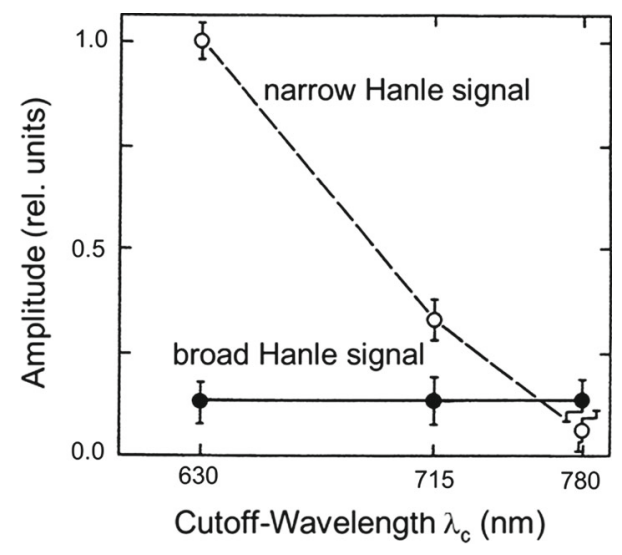

Fig. 9 The signal amplitude of the narrow and the broad Hanle signal measured in the detection windows with the cutoff-wavelength $\lambda_{\mathrm{c}}=630 \mathrm{~nm}, 715 \mathrm{~nm}$, and $780 \mathrm{~nm}$

because the signal amplitude $S_{n}$ decreases strongly with $\lambda_{c}$. We associate this change of $S_{n}$ with $\lambda_{c}$ to a change in the degree of polarization of the fluorescence light of $\mid \mathrm{e}, 2>$. The degree of polarization of the fluorescence light depends on the symmetry of the molecule in the upper and the lower state of the radiative transition in the fluorescence decay. In Eq. 9, we introduce the quantity $\mathrm{w}=\mathrm{w}_{\mathrm{P}}-\mathrm{w}_{\mathrm{Q}}+\mathrm{w}_{\mathrm{R}}$, where the subscripts $\mathrm{P}, \mathrm{Q}$, and $\mathrm{R}$ stand for $\mathrm{P}-, \mathrm{Q}-$, and R-branch transitions in the fluorescence decay [27]. The degree of polarization of the fluorescence light in Q-branch emission $(\Delta \mathrm{F}=0)$ has the opposite sign to the degree of polarization of the fluorescence light in $\mathrm{P}$ - and $\mathrm{R}$-branch emission $(\Delta \mathrm{F}= \pm 1)$ [47]. If we identify $\mid \mathrm{e}, 1>$ with the $\mathrm{C}_{2 \mathrm{v}}$ symmetric state ${ }^{2} \mathrm{~B}_{2}$, we expect $\mathrm{w}_{\mathrm{Q}} \approx 0$ and $\mathrm{w} \approx \mathrm{w}_{\mathrm{P}}+\mathrm{w}_{\mathrm{R}} \approx$ constant, because the radiative decay of $\mid \mathrm{e}, 1>$ follows the selection rules for a near prolate symmetric top $[21,22]$. Therefore, $S_{b}$ is independent of $\lambda_{c}$. On the other hand, $\mathrm{S}_{\mathrm{n}}$ has a decreasing $\mathrm{w}$ versus $\lambda_{\mathrm{c}}$ because the contribution of Q-branch transitions in w increases with higher energy in the final state of the fluorescence decay. If we assume that $\mid \mathrm{e}, 2>$ does not have $\mathrm{C}_{2 \mathrm{v}}$ symmetry, then the final state in the fluorescence decay will strongly determine the degree of polarization of the fluorescence light. For a molecule having no symmetry in the initial and final state of the fluorescence decay, there is no way to discriminate between $\mathrm{w}_{\mathrm{P}}, \mathrm{w}_{\mathrm{Q}}$, and $\mathrm{w}_{\mathrm{R}}[48]$. We expect $\mathrm{w}=\mathrm{w}_{\mathrm{P}}-\mathrm{w}_{\mathrm{Q}}+\mathrm{w}_{\mathrm{R}} \approx 0$. Therefore, the dependence of $\mathrm{S}_{\mathrm{n}}$ on $\lambda_{\mathrm{c}}$ suggests, that the transition $\mid \mathrm{e}, 1>\rightarrow \mathrm{e}, 2>$ represents a change from the $\mathrm{C}_{2 \mathrm{v}}$ symmetric state ${ }^{2} \mathrm{~B}_{2}$ to an asymmetric configuration of the molecule.

The broad and the narrow Hanle signal reveal the existence of the states $\mid \mathrm{e}, 1>$ and $\mid \mathrm{e}, 2>$ in the transition $|\mathrm{e}, 1>\rightarrow| \mathrm{e}, 2>$. The state $\mid \mathrm{e}, 1>$ has a lifetime $\tau_{0} \approx 3 \mu \mathrm{s}$ which is not determined by radiative decay. The ratio of the amplitudes of both Hanle signals reveals that both states have about the same radiative lifetime $\tau_{\mathrm{R}} \approx 35 \mu \mathrm{s}$ in agreement time resolved fluorescence decay measurements, which reveal single exponential radiative decay of the excited state. Both states differ in the degree of polarization of the fluo- 
rescence light indicating that both states have different symmetries. We note that measurements of an opticalrf double resonance signal associated with the state e, $1>$ were not successful, because these measurements are strongly perturbed by the broad rf-resonances discussed in Sect. 5. In Sect. 4 all $\mathrm{T}_{\mathrm{L}}$ values are based on the adjustment to minimum absorption according to Fig. 3.

\section{Enhanced absorption resonances}

The narrow and the broad Hanle signal as well as the optical-rf double resonance signal discussed in Sects. 3 and 4 represent a resonant change in the degree of polarization of the fluorescence light. The width of these signals is independent of the light intensity and of the transit time $\mathrm{T}_{\mathrm{L}}$. In this section we report on two resonances in the fluorescence intensity $\mathrm{F}$, the "o-resonance" and the "broad rf-resonance". We mentioned both resonances in Sect. 3. Figure 4 depicts the $\sigma$-resonance and the narrow Hanle signal. The broad rfresonance is mentioned in the discussion of the opticalrf double resonance experiments. We investigate both resonances using the one-light-beam setup depicted in Fig. 2a and the "adjustment to minimum absorption" of the divergence of the light beam according to Fig. 3. The minimum of the fluorescence intensity $\mathrm{F}$ in Fig. 3 indicates that the optical field induces in the molecule many cycles of absorption and induced emission in the transition $|\mathrm{g}, 1>\leftrightarrow| \mathrm{e}, 1>$. Therefore, the effective absorption is low, and $\mathrm{F}$ has a minimum. A rf-field resonant in the magnetic sublevels of $\mid \mathrm{g}, 1>$ or of $\mid \mathrm{e}, 1>$ but also a static magnetic field B (perpendicular to the linear polarization of the light beam) perturb the optical coherence in the transition $|\mathrm{g}, 1>\leftrightarrow| \mathrm{e}, 1>$ and reduce the number of cycles of absorption and induced emission in this transition. This results in an increase of the fluorescence intensity $\mathrm{F}$ by enhanced absorption. The broad rf-resonance and the $\sigma$-resonance are both enhanced absorption resonances, have a width independent of the light intensity, but strongly dependent on the transit time $\mathrm{T}_{\mathrm{L}}$ of the molecules through the light beam. For vanishing transit time broadening the widths of both resonances yield the time constant $\tau_{0} \approx 3 \mu \mathrm{s}$ as we will show.

We discuss the experimental results of the broad rfresonance first because we have a model for this resonance (Ref. [46] and Appendix A). In the experiment, the static magnetic field $\mathrm{B}$ is directed along the $\mathrm{x}$-axis parallel to the molecular beam and the rf-field $B_{1}$ is linearly polarized along the $\mathrm{y}$-axis. The earth magnetic field is compensated. The laser light has linear polarization $\mathbf{e}_{\mathrm{x}}$ parallel to the B-field ( $\pi$-polarized excitation, $\Delta \mathrm{m}=0$ transitions), and the fluorescence intensity $\mathrm{F}$ is detected in the detection window $630 \mathrm{~nm}\langle\lambda>$ $850 \mathrm{~nm}$ (see Sect. 2) along the z-axis by one photodetector (no polarizer in the detection pass). The measured quantity is $\mathrm{S}=\left(\mathrm{F}-\mathrm{F}_{0}\right) / \mathrm{F}_{0}$ versus the magnetic field $\mathrm{B}$ with $\mathrm{F}_{0}=\mathrm{F}$ when the magnetic field $\mathrm{B}$ is off resonance.

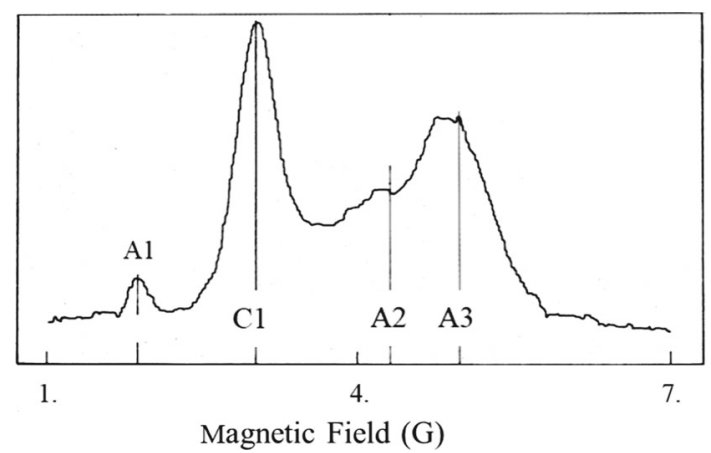

Fig. 10 Magnetic resonance spectrum detected in the fluorescence intensity $\mathrm{F}$ versus the magnetic field $\mathrm{B}$ at the rffrequency $\nu_{\mathrm{rf}}=2.5 \mathrm{MHz}$ of $\mathrm{B}_{1}$ with the laser light tuned to line $1 \mathrm{R}$

Figure 10 depicts a resonance spectrum versus the magnetic field $\mathrm{B}$ at the rf-frequency $\nu_{\mathrm{rf}}=2.5 \mathrm{MHz}$ of $\mathrm{B}_{1}$ with the laser light tuned to line $1 \mathrm{R}$, the aperture width $\mathrm{d}=3 \mathrm{~mm}$, and the light intensity $\mathrm{I}=15 \mathrm{~mW} / \mathrm{mm}^{2}$. The resonances are an increase of $\mathrm{F}$ up to $5 \%$ whenever B satisfies the resonance condition in Eq. 3a. With A1, A2, A3, and C1 we indicate the expected magnetic field values of resonance for the g-factors involved in the absorption line $1 \mathrm{R}$ (see Fig. 1). The resonances in Fig. 10 have a large width and overlap strongly. The overlapping of the resonances seems to shift the resonance peaks away from the expected position. However, with a larger aperture width $\mathrm{d}$, the resonances are better resolved and show good agreement (precision 20\%) between the expected (Eq. 2) and the observed positions of the resonances. We note that the same measurement as in Fig. 10 except with an aperture width $\mathrm{d}=1 \mathrm{~mm}$ results in a resonance spectrum, in which all resonances of Fig. 10 grow together to one broad unresolved resonance structure (see Fig. 3 in Ref. [35]). Larger values of the magnetic field $B$ and of the frequency $\nu_{\mathrm{rf}}$ do not improve the resolution because the resonance condition in Eq. 3a requires $B<3 \mathrm{G}$ (see Sect. 2). The resonances A2 and A3 in Fig. 10 are strongly broadened due to the non-equidistant Zeeman splitting of the magnetic sublevels.

We choose the resonance $\mathrm{C} 1$ for the investigation of the broad rf-resonance using $\nu_{\mathrm{rf}}=1.4 \mathrm{MHz}$ with $\mathrm{B}=1.5 \mathrm{G}$ at the resonance $\mathrm{C} 1$. The experimental results reveal that the resonance $\mathrm{C} 1$ has a Lorentzian line-shape for light intensities $\mathrm{I}<10 \mathrm{~mW} / \mathrm{mm}^{2}$, for aperture widths $\mathrm{d}<10 \mathrm{~mm}$, and for low power of the rf-field [35]. For these values of $\mathrm{I}$ and $\mathrm{d}$, and for low power of the rf-field, the resonance $\mathrm{C} 1$ has an amplitude proportional to I and the power of the rf-field, a width independent of I and the power of the rf-field and agrees well with the model of the broad rf-resonance discussed in Refs. [35,46] (see Appendix A). The model predicts the resonance signal $\mathrm{S}_{\mathrm{B}}$ 


$$
S_{B}=\frac{\left[1-\exp \left(-\Gamma_{b c} T_{L}\right)\right]}{\Gamma_{b c}\left(\Gamma_{a c}+b_{L}\right)} \cdot \frac{M}{\left[\left(\omega_{a}-\omega_{r f}\right)^{2}+\left(\Gamma_{a c}+b_{L}\right)^{2}\right]}
$$

$$
M=\sum_{m, r} A_{m}\left|G_{a b}^{m m}\right|^{2}\left|B_{a a}^{m, m+r}\right|^{2}
$$

$\mathrm{S}_{\mathrm{B}}$ represents the broad rf-resonance associated with the optical transition $|\mathrm{g}, 1>\leftrightarrow| \mathrm{e}, 1>$ between a hfslevel of the lower state and a hfs-level of the upper state, and with rf-resonance in $\mid \mathrm{g}, 1>$. As in Sect. 3, we introduce $|\mathrm{g}, 1, \mathrm{~m}\rangle=|\mathrm{a}, \mathrm{m}\rangle$ and $|\mathrm{e}, 1, \mathrm{~m}\rangle=|\mathrm{b}, \mathrm{m}\rangle$ for the lower and the upper state of the optical transition with the Rabi frequency $G_{a b}^{m m}$ of the optical transition $|\mathrm{g}, 1, \mathrm{~m}>\leftrightarrow| \mathrm{e}, 1, \mathrm{~m}>$ and with $\left|G_{a b}^{m m}\right|^{2}$ being proportional to the light intensity I. The rf-resonance in the lower state is represented by the Rabi frequency $B_{a a}^{m, m+r}$ of the rf-transition $|\mathrm{g}, 1, \mathrm{~m}>\leftrightarrow| \mathrm{g}, 1, \mathrm{~m}+\mathrm{r}>$ with $\mathrm{r}= \pm 1$ and with $\left|B_{a a}^{m, m+r}\right|^{2}$ being proportional to the power of the rf-field $\mathrm{B}_{1}$. Moreover, $A_{m}=$ $b_{m} /\left(\sum_{n} b_{n}\right)$ is the occupation probability in the magnetic sublevels $|\mathrm{e}, 1, \mathrm{~m}\rangle=|\mathrm{b}, \mathrm{m}\rangle, \omega_{\mathrm{a}}$ is the Zeeman splitting in the lower state, $\omega_{\mathrm{rf}}=2 \pi \nu_{\mathrm{rf}}$ is the rf-frequency, $b_{L}$ is the spectral width of the laser light as seen by the molecules during the transit time $\mathrm{T}_{\mathrm{L}}$ through the light beam, and $\Gamma_{\mathrm{bc}}$ and $\Gamma_{\mathrm{ac}}$ are phenomenologically introduced rate constants associated with the transition $|\mathrm{e}, 1>\rightarrow| \mathrm{e}, 2>$.

The experimental resonance $\mathrm{C} 1$ is the sum of all signals $\mathrm{S}_{\mathrm{B}}$ associated with the transitions $|\mathrm{g}, 1>\leftrightarrow| \mathrm{e}, 1>$ from the two hfs-levels $\mid \mathrm{N}=0, \mathrm{~J}=1 / 2, \mathrm{~F}=3 / 2>$ and $\mathrm{N}=0, \mathrm{~J}=1 / 2, \mathrm{~F}=1 / 2>$ in the lower state of line $1 \mathrm{R}$ (see Fig. 1) to all hfs-levels of the upper state on line $1 R$. These signals differ only in the quantity $M$ because the rate constants $\Gamma_{\mathrm{bc}}$ and $\Gamma_{\mathrm{ac}}$ are the same on all hfslevels belonging to the same vibrational level. We determine $\Gamma_{\text {ac }}$ from measurements of the width (see below) and subsequently $\Gamma_{\mathrm{bc}}$ from the dependence of the signal amplitude $\mathrm{S}_{\mathrm{B}}$ in Eq. 10 on the transit time $\mathrm{T}_{\mathrm{L}}$. Figure 5 in Ref. [35] depicts measurements of the amplitude of the resonance $\mathrm{C} 1$ versus the aperture width $\mathrm{d}=\mathrm{uT}_{\mathrm{L}}$ and a fit of the amplitude of $\mathrm{S}_{\mathrm{B}}$ in Eq. 10 to the experimental results. This fit yields $\Gamma_{\mathrm{bc}}=0.31 \mathrm{MHz}$ and therefore $1 / \Gamma_{\mathrm{bc}}=3.2 \mu \mathrm{s}$ with an estimated error of $20 \%$.

The width (HWHM) of the broad rf-resonance $\mathrm{S}_{\mathrm{B}}$ in Eq. 10 is $\Delta\left(\omega_{\mathrm{a}}-\omega_{\mathrm{rf}}\right)=\Gamma_{\mathrm{ac}}+\mathrm{b}_{\mathrm{L}}$. With the Zeeman splitting $\omega_{\mathrm{a}}=|\mathrm{g}|\left(\mu_{\mathrm{B}} / \hbar\right) \mathrm{B}$ in the ground state $\mid \mathrm{g}, 1>$, the experimentally determined width $\Delta \mathrm{B}_{\mathrm{rf}}$ is given by $\Delta\left(\omega_{\mathrm{a}}-\omega_{\mathrm{rf}}\right)=\left(\mu_{\mathrm{B}} / \hbar\right)|\mathrm{g}| \Delta \mathrm{B}_{\mathrm{rf}}$. Figure 11 depicts the measured width $\Delta \mathrm{B}_{\mathrm{rf}}$ (HWHM) of the broad rf-resonance $\mathrm{C} 1$ versus $1 / \mathrm{d}$ at the light intensity $\mathrm{I}=2.5 \mathrm{~mW} / \mathrm{mm}^{2}$. The results are represented by

$$
\begin{aligned}
& \Delta \mathrm{B}_{\mathrm{rf}}=45 \mathrm{mG}+(210 / \mathrm{d}) \mathrm{mG} \mathrm{mm} \\
& \left(\mu_{\mathrm{B}} / \hbar\right)|\mathrm{g}| \Delta \mathrm{B}_{\mathrm{rf}}=\Gamma_{\mathrm{ac}}+\mathrm{b}_{\mathrm{L}} \\
& \quad=0.26(\mu \mathrm{s})^{-1}+2.0\left(\mathrm{~T}_{\mathrm{L}}\right)^{-1}
\end{aligned}
$$

with $|\mathrm{g}|=0.667, \mathrm{~T}_{\mathrm{L}}=\mathrm{d} / \mathrm{u}$, and $\mathrm{u}=610 \mathrm{~ms}^{-1}$. The measurements are restricted to a narrow range of values of $1 / \mathrm{d}$ due to the overlapping of the resonance $\mathrm{C} 1$ with other resonances (see Fig. 10) and due to the condition $\mathrm{d}<10 \mathrm{~mm}$ (see above). We obtain $1 / \Gamma_{\mathrm{ac}}=3.9 \mu \mathrm{s}$ with an estimated error of $20 \%$. The same figure also depicts measurements on the $\sigma$-resonance, which we will discuss next.

Figure 4 in Sect. 3 depicts the $\sigma$-resonance in various fluorescence detection arrangements, e.g. in the arrangement $[\sigma \uparrow \pi \downarrow]$. Like the broad rf-resonance, the $\sigma$-resonance is primarily a change of the total fluorescence intensity $\mathrm{F}$. The signal strength in the detection arrangement $[\sigma \uparrow(\pi-\sigma) \downarrow]$ is about a factor of 10 smaller than in the arrangement $[\sigma \uparrow \pi \downarrow]$, which we use for the study of the $\sigma$-resonance [34]. The asymmetry in the $\pi$ - and $\sigma$ - fluorescence detection is discussed in Ref. [34]. The $\sigma$-resonance has a Lorentzian line shape, a width independent of the light intensity I (Fig. 4 of Ref. [34]), and a signal strength proportional to I for values of $\mathrm{d}$ and I with $\mathrm{d} \cdot \mathrm{I}<50 \mathrm{~mW} / \mathrm{mm}$ (for details see Ref. [34]). We restrict the investigations of the $\sigma$-resonance to this range of parameter values $\mathrm{d}$ and I. Figure 11 depicts besides the width $\Delta \mathrm{B}_{\mathrm{rf}}$ of the broad rf-resonance also the widths (HWHM) of the $\sigma$ resonances $\Delta \mathrm{B}_{\sigma 1}$ on line 1 and $\Delta \mathrm{B}_{\sigma 1 \mathrm{R}}$ on line $1 \mathrm{R}$. The ratio $\Delta \mathrm{B}_{\sigma 1} / \Delta \mathrm{B}_{\sigma 1 \mathrm{R}}=1.58 \pm 0.05$ is independent of the aperture width $\mathrm{d}$.

The $\sigma$-resonance depends on the g-factors in the upper and the lower state of the laser induced transition [34]. Several optical transitions are possible between the hfs-components in the lower and the upper finestructure level, and all may contribute with different strength to the $\sigma$-resonance (see Fig. 1). On line $1 \mathrm{R}$, the transition $|\mathrm{N}=0, \mathrm{~J}=1 / 2, \mathrm{~F}=3 / 2>\leftrightarrow| \mathrm{N}=1$, $\mathrm{J}=3 / 2, \mathrm{~F}=5 / 2>$ is the strongest as the spectrum of the broad rf-resonances in Fig. 10 reveals. This transition involves the g-factors $\mathrm{g}_{\mathrm{a}}=0.667$ in the lower state and $\mathrm{g}_{\mathrm{b}}=0.400$ in the upper state. We introduce the effective g-factor $\mathrm{g}_{1 \mathrm{R}}=\mathrm{g}_{\mathrm{a}}+\mathrm{g}_{\mathrm{b}}=1.067$ for the $\sigma$-resonance on line $1 \mathrm{R}$. Similarly, the spectrum of the broad rf-resonances on line 1 (see Fig. 2 of Ref. [35]) reveals the transition $\mid \mathrm{N}=2, \mathrm{~J}=5 / 2$, $\mathrm{F}=7 / 2>\leftrightarrow \mid \mathrm{N}=1, \mathrm{~J}=3 / 2, \mathrm{~F}=5 / 2>$ as strongest transition. This transition yields the effective g-factor $\mathrm{g}_{1}=\mathrm{g}_{\mathrm{a}}+\mathrm{g}_{\mathrm{b}}=0.286+0.400=0.686$ on line 1 . The ratio $\mathrm{g}_{1 \mathrm{R}} / \mathrm{g}_{1}=1.56$ agrees well with the ratio $\Delta \mathrm{B}_{\sigma 1} / \Delta \mathrm{B}_{\sigma 1} \mathrm{R}$. This gives $\left|g_{1}\right| \Delta B_{\sigma 1}=\left|g_{1 R}\right| \Delta B_{\sigma 1 R}$. With the experimental result $\Delta B_{\sigma 1 \mathrm{R}}=34 \mathrm{mG}+(140 / \mathrm{d}) \mathrm{mG} \mathrm{mm}$ and with the same relation (Eq. 11) as for the broad rfresonance we obtain [34]

$$
\begin{aligned}
\left(\mu_{\mathrm{B}} / \hbar\right)\left|\mathrm{g}_{1}\right| \Delta \mathrm{B}_{\sigma 1} & =\left(\mu_{\mathrm{B}} / \hbar\right)\left|\mathrm{g}_{1 \mathrm{R}}\right| \Delta \mathrm{B}_{\sigma 1_{\mathrm{R}}} \\
& =\Gamma_{\mathrm{ac}}+\mathrm{b}_{\mathrm{L}} \\
& =0.318(\mu \mathrm{s})^{-1}+2.15\left(\mathrm{~T}_{\mathrm{L}}\right)^{-1}
\end{aligned}
$$

and $1 / \Gamma_{\mathrm{ac}}=3.1 \mu \mathrm{s}$ with an estimated error of $20 \%$. As reported in Ref. [34], the measurements of the $\sigma$ resonance on other lines in the $593 \mathrm{~nm}$ band agree within $30 \%$ with the results on lines 1 and $1 \mathrm{R}$. Measurements of the Hanle signal and of the $\sigma$-resonance are also performed at $\lambda_{\text {ex }}=514 \mathrm{~nm}$ with use of a $\mathrm{cw}$ single-mode Ar-ion laser and reveal a coherence decay 


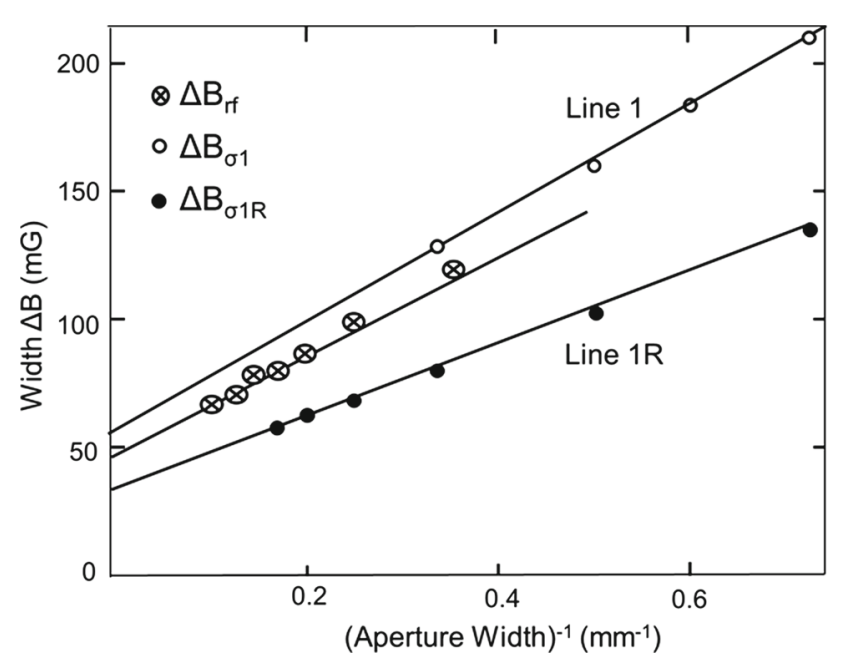

Fig. 11 The width $\Delta \mathrm{B}_{\mathrm{rf}}$ (HWHM) of the broad rfresonance $\mathrm{C} 1$ and the widths (HWHM) of the $\sigma$-resonances $\Delta \mathrm{B}_{\sigma 1}, \Delta \mathrm{B}_{\sigma 1 \mathrm{R}}$ on line 1 and line $1 \mathrm{R}$ versus $1 / \mathrm{d}$ where $\mathrm{d}$ is the aperture width

time $\tau_{\mathrm{c}}=27 \pm 5 \mu \mathrm{s}$ in agreement with the radiative lifetime $\tau_{\mathrm{R}}$ and the value $1 / \Gamma_{\mathrm{ac}}=2.3 \pm 1 \mu \mathrm{s}$ [34].

The experiments reveal $1 / \Gamma_{\mathrm{ac}}=1 / \Gamma_{\mathrm{bc}}=\tau_{0} \approx 3 \mu \mathrm{s}$ within the uncertainty of the results. All measured values for $\tau_{0}$ are within $2 \mu \mathrm{s}<\tau_{0}<4 \mu \mathrm{s}$. We assign the strong dependence of the width of both resonances on the parameter $1 / \mathrm{d}$ to transit time broadening of the spectral width seen by the molecules. However, the expected value (HWHM) of transit time broadening is $\Delta \omega=2.79 / \mathrm{T}_{\mathrm{L}}$ with $\mathrm{T}_{\mathrm{L}}=\mathrm{d} / \mathrm{u}$ (see Ref. [49]), whereas the measurements reveal values close to $\Delta \omega=2.1 / \mathrm{T}_{\mathrm{L}}$ $[34,35]$. The results in Sect. 6 suggest that the spectral width of the light beam seen by the molecules may also have a component proportional to $\mathrm{d}$ which is due to laser frequency jitter during the time of flight through the light beam. This component is not considered in the evaluation of the data in Fig. 11.

\section{The width of absorption lines}

Sections 3-5 report on experiments in which one light beam (L1) induces in the molecule the optical transition $|\mathrm{g}, 1>\leftrightarrow| \mathrm{e}, 1>$ and the fluorescence decay is detected. The optical transition $|\mathrm{g}, 2>\leftrightarrow| \mathrm{e}, 2>$ is not directly involved. The present section reports on an experiment in the two-light-beams setup depicted in Fig. 2b. Here the transition $|\mathrm{g}, 2>\leftrightarrow| \mathrm{e}, 2>$ is important. The light of a single mode cw dye-laser is split into the two beams L1 and L2 with L1 interacting with the molecules first and primarily in the transition $|\mathrm{g}, 1>\leftrightarrow| \mathrm{e}, 1>$, and with L2 interacting with the molecules after a time-of-flight $\mathrm{T} \leq \tau_{\mathrm{R}}$ and primarily in the transition $|\mathrm{g}, 2>\leftrightarrow| \mathrm{e}, 2>$. The experiments are described in detail in Ref. [37].

Figure 12 depicts a measurement of the fluorescence intensity $\mathrm{F}$ versus the angle $\alpha$ in Fig. 2b. However, instead of $\alpha$ we use $\delta v=v(u / c) \alpha=1.03 \alpha \mathrm{MHz} / \mathrm{mrad}$ with $u=610 \mathrm{~m} / \mathrm{s}$ and $\mathrm{c}$ the velocity of light, where $\delta v$ is the frequency difference of L1 and L2 as seen by the molecules if $\alpha$ is varied [37]. At $\delta \nu=0$, i.e. $\alpha=0$, the fluorescence intensity $\mathrm{F}$ has a minimum, the light beams L1 and L2 are parallel to each other, separated by the gap width s. The inset A in Fig. 12 shows the original signal detected by the photomultiplier before the signal processing, and the inset B depicts the intersection of L1 and L2 with the effusive molecular beam. The change of $\alpha$ shifts the intersection of L2 with the molecular beam along the molecular beam axis from a region of higher density $(\alpha, \delta \nu<0)$ to a region of lower density $(\alpha, \delta \nu>0)$. This causes the oblique background of the measured signal in the inset A of Fig. 12. If L1 is off, there is no signal at $\delta \nu=0$ but still the oblique background. We subtract this background in the processing of the data for the evaluation of the resonance signal and call the signal depicted in Fig. 12 "v-resonance". In the measurement in Fig. 12 the laser light is tuned (at $\alpha=0$ ) to line $1 \mathrm{R}$ and both light beams have linear polarization parallel to the $\mathrm{x}$-axis (the molecular beam axis). There is no rf- field $B_{1}$ and the earth magnetic field is compensated. Experiments are performed with and without a static magnetic field B directed parallel to the $\mathrm{x}$-axis. The $\mathrm{v}$-resonance has no Lorentzian shape. It is better, but not exactly, fitted by a Gaussian shape. The width and amplitude depend on the divergence of L1 and L2. The width has a minimum and the amplitude a maximum if we use the "adjustment to minimum absorption" for the beam divergence of L1 and L2 (see Fig. 3). This adjustment of the light beams allows reproducible measurements with a precision of better than $5 \%$. Measurements of the $v$-resonance at magnetic fields $\mathrm{B}$ between $\mathrm{B}=0$ and $\mathrm{B}=50 \mathrm{G}$ reveal no effect of $B$ on the amplitude and width of the $\nu$ resonance for $\mathrm{B}$ parallel to the $\mathrm{x}$-axis ( $\pi$-excitation). This is different in the case of $\sigma$-excitation [37]. We measure the $\nu$-resonance on all investigated (about 20) absorption lines in the $593 \mathrm{~nm}$ band. The width is the same on all lines (within a precision of $5 \%$ ). Also, the signal strength is approximately the same. In Ref.[37] we discuss the effect of the velocity distribution of the molecules along the molecular beam axis on this resonance and conclude that it may affect the shape and the width by less than $20 \%$. In the following we describe the properties of the $\nu$-resonance measured on line $1 R$ in detail.

We investigate the width (FWHM) $\Delta v$ and the amplitude $\mathrm{A}_{v}$ of the quantity $\mathrm{A}=\left(\mathrm{F}-\mathrm{F}_{0}\right) / \mathrm{F}_{0}$ where $\mathrm{F}_{0}=\mathrm{F}$ if $\delta \nu$ is far off-resonance. In Ref. [37] (Figs. 68 ), we report on measurements of $\Delta v$ and $A_{v}$ versus the light intensity I of L1 and L2, versus the gap width $\mathrm{s}$ between L1 and L2, and versus the aperture width d. The width $\Delta v$ is independent of the light intensity I (between 0.2 and $10 \mathrm{~mW} / \mathrm{mm}^{2}$ ), whereas $\mathrm{A}_{\nu}$ exhibits strong saturation for $\mathrm{I}>3 \mathrm{~mW} / \mathrm{mm}^{2}$. The results of the measurements versus $\mathrm{s}$ and $\mathrm{d}$ are summarized by the following equations:

$$
\Delta v=\Delta v_{0}+\Delta v_{t}+\Delta v_{j}
$$




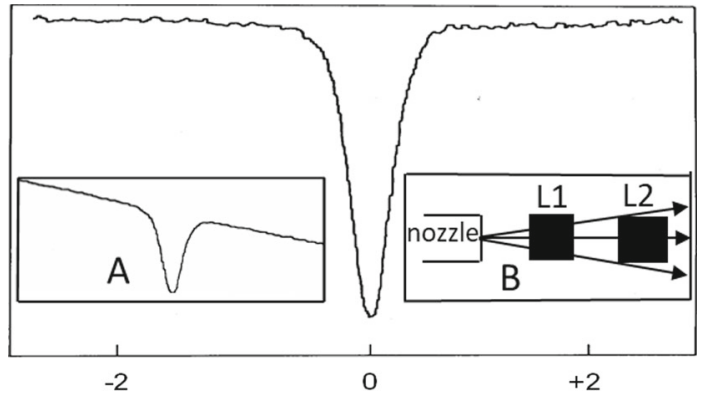

Frequency Difference ōv $(\mathrm{MHz})$

Fig. 12 The fluorescence intensity $F$ in the two-lightbeams set-up versus the angle $\alpha$ in Fig.2b. We use $\delta v=$ $\nu(\mathrm{u} / \mathrm{c}) \alpha=1.03 \alpha \mathrm{MHz} / \mathrm{mrad}$ with $\delta \nu$ the frequency difference of L1 and L2 as seen by the molecules. Inset A shows the original signal before the processing of the signal and inset B depicts the intersection of L1 and L2 with the effusive molecular beam

$$
\begin{aligned}
& \Delta v_{0}=(0.13 \pm 0.05) \mathrm{MHz} \\
& \Delta v_{\mathrm{t}}=(0.87 \pm 0.05)\left(\mathrm{T}_{\mathrm{L}}\right)^{-1} \\
& \Delta v_{\mathrm{j}}=(7.6 \pm 1.0) 10^{9}\left(\mathrm{~T}_{\mathrm{s}}+\mathrm{T}_{\mathrm{L}}\right)(\mathrm{Hz})^{2} \\
& \mathrm{~A}_{\nu}=\mathrm{A}_{0} \exp \left[-\left(\mathrm{T}_{\mathrm{s}}+\mathrm{T}_{\mathrm{L}}\right)\left(\tau_{\mathrm{s}}\right)^{-1}\right], \\
& \tau_{\mathrm{s}}=(22 \pm 2) \mu \mathrm{s}
\end{aligned}
$$

Here we use $\left(T_{S}+T_{L}\right)=[(s+d) / u]$. The width $\Delta v$ of the $\nu$-resonance comprises three terms (see Eq. 13a), with $\Delta v_{\mathrm{t}}$ representing the transit time broadening in good agreement with theory [49], and with $\Delta v_{\mathrm{j}}$ representing laser frequency jitter during the time of flight $\left(\mathrm{T}_{\mathrm{S}}+\mathrm{T}_{\mathrm{L}}\right)$ between the centres of L1 and L2. A term like $\Delta v_{j}$ is expected for a mean square displacement of the laser cavity length due to spontaneous fluctuations during the "observation time" $\left(\mathrm{T}_{\mathrm{s}}+\mathrm{T}_{\mathrm{L}}\right)$ [50]. Finally, we associate $\Delta \nu_{0}$ with the absorption width of the state $\mid \mathrm{e}, 1>$. The model of the space-coupled molecule (Sect. 1) predicts an absorption width (FWHM) $\Delta \nu_{0}=\left(\pi \tau_{0}\right)^{-1}$ determined by the time constant $\tau_{0}$ and not by the radiative lifetime $\tau_{R}$. The result in Eq. $13 \mathrm{~b}$ gives $\tau_{0}=$ $2.4 \pm 0.7 \mu \mathrm{s}$, which is consistent with the measurements of $\tau_{0}$ reported in Sects. 4 and 5 . The width $\Delta \nu_{0}$ is smaller than the hfs-splitting in the ground and excited state of $\mathrm{NO}_{2}$ (see Sect. 2) and at large magnetic field ( $\mathrm{B} \approx 50 \mathrm{G}$ where the hyperfine structure is decoupled, see Sect. 2) smaller than the splitting of magnetic sublevels. Therefore, for a given molecule, the light beams L1 and L2 induce a transition between a single hfs-level of the lower and a single hfs-level of the upper state or at large values of $\mathrm{B}$ transitions between isolated magnetic sublevels in the lower and the upper state. Due to the residual Doppler width in the molecular beam the laser light induces several of these two-level transitions on line 1R (see Fig. 1). Thus the $v$-resonance is a sum of several independent signals of which each signal is centred at $\delta v=0$ with the same components $\Delta \nu_{0}, \Delta v_{t}$, and $\Delta v_{\mathrm{j}}$ of the width $\Delta v$.

The amplitude $\mathrm{A}_{v}$ (see Eq. 13e) decreases exponentially versus the time-of-flight $\left(T_{S}+T_{L}\right)$ between the centres of L1 and L2 with a time constant $\tau_{\mathrm{s}}=$ $(22 \pm 2) \mu \mathrm{s}$ which is smaller than the radiative lifetime $\tau_{\mathrm{R}}=35 \mu \mathrm{s}$ of the excited state. The inset B in Fig. 12 explains the result $\tau_{s}<\tau_{R}$. The number of excited molecules interacting with L2 decreases stronger than the exponential decay with the radiative lifetime $\tau_{\mathrm{R}}$ predicts, because the number of molecules which pass L2 after excitation in L1 decreases with the gap width s between L1 and L2 in the effusive molecular beam. In Ref. [37] we study this geometrical effect by performing a similar experiment using an $\mathrm{I}_{2}$ molecular beam. The results of this experiment reveal that the geometrical effect causes an effective shortening of the measured lifetime by about $30 \%$. Taking this geometrical effect in account we conclude that $A_{v}$ decreases versus the time of flight $\left(\mathrm{T}_{\mathrm{S}}+\mathrm{T}_{\mathrm{L}}\right)$ in agreement with the radiative lifetime $\tau_{R}$. This result is unusual and is discussed in detail in Ref. [37]. However, this result agrees with the model of the space-coupled molecule represented by Eqs. 1a$1 c$.

The laser-induced transitions $|\mathrm{g}, 1>\leftrightarrow| \mathrm{e}, 1>$ and $|\mathrm{g}, 2>\leftrightarrow| \mathrm{e}, 2>$ are very different. The experimental results reported in Sects. 2-5 reveal that a molecule experiences many cycles of absorption and stimulated emission in the optical transition $|\mathrm{g}, 1>\leftrightarrow| \mathrm{e}, 1>$ before it evolves into $\mid \mathrm{e}, 2>$. This is evident from the adjustment of the light beam to minimum absorption in Sect. 2 (Fig. 3), from the inversion effect in Sect. 3 and from the enhanced absorption resonances in Sect. 5. Therefore, the efficiency to populate $\mid \mathrm{e}, 2>$ in the transition $|\mathrm{g}, 1>\leftrightarrow| \mathrm{e}, 1>$ is low. On the other hand, the optical transition $|\mathrm{g}, 2>\leftrightarrow| \mathrm{e}, 2>$ enables no absorption but only stimulated emission from $\mid \mathrm{e}, 2>$ to $\mid \mathrm{g}, 2>$, because the non-radiative transition $|\mathrm{g}, 2>\rightarrow| \mathrm{g}, 1>$ is very fast and depopulates $\mid \mathrm{g}, 2>$ within few vibrational periods. The low effective population rate of $\mid \mathrm{e}, 2>$ via the transition $|\mathrm{g}, 1>\leftrightarrow| \mathrm{e}, 1>$ and a high rate of induced emission in the transition $|\mathrm{g}, 2>\leftrightarrow| \mathrm{e}, 2>$ explain the strong saturation (about $50 \%$ at $\mathrm{I}=10 \mathrm{~mW} / \mathrm{mm}^{2}$ ) of the amplitude $A_{v}$ versus the light intensity reported above. The state $\mid \mathrm{e}, 2>$ is much faster depopulated by stimulated emission than populated via the non-radiative transition $|\mathrm{e}, 1>\rightarrow| \mathrm{e}, 2>$. The exponential decrease of the amplitude $A_{v}$ versus the time of flight $\left(T_{S}+T_{L}\right)$ is a direct consequence of the low effective absorption rate in the transition $|\mathrm{g}, 1>\leftrightarrow| \mathrm{e}, 1>$ (see also discussion in [37]).

We identify the width $\Delta \nu_{0}$ of the $\nu$-resonance with the width of the $\mathrm{NO}_{2}$ absorption line, i.e. with the spectral width of $\mid \mathrm{e}, 1>$. The width $\Delta \nu_{0} \approx 130 \mathrm{kHz}$ is much larger than the natural linewidth $\left(\pi \tau_{\mathrm{R}}\right)^{-1} \approx 9 \mathrm{kHz}$ of $\mid \mathrm{e}, 2>$. Obviously $\mid \mathrm{e}, 2>$ is having a level energy somewhere within the width of $\mid \mathrm{e}, 1>$. The uncertainty of the level energy of $\mid e, 2>$ is not only given by the radiative width. In $\mid \mathrm{e}, 2>$ the molecule is still coupled to the extra compactified dimensions of space. We assign the near Gaussian shape of the $\nu$-resonance to the distribution of the level energies of $\mid \mathrm{e}, 2>$ within the width of $|\mathrm{e}, 1\rangle$. As reported above, the width of the " $\nu$-resonance" is independent of the light intensity although the signal amplitude reveals strong saturation 
behaviour. Obviously, the light intensity may broaden the single $\mid e, 2>$ levels but not the distribution of the level energies within the width of $\mid e, 1>$.

\section{Discussion and conclusion}

In this paper, we report on experimental results on the triatomic molecule $\mathrm{NO}_{2}$. The key- result is the inversion effect described in Sects. 3 and 4. This effect reveals that the collision-free molecule optically excited into a state $\mid \mathrm{e}, 1>\left(\right.$ a hfs-level) undergoes within a time $\tau_{0} \approx 3 \mu \mathrm{s}$ an irreversible non-radiative transition from $\mid \mathrm{e}, 1>$ to a state $\mid \mathrm{e}, 2>$. Both $\mid \mathrm{e}, 1>$ and $\mid \mathrm{e}, 2>$ have about the same radiative lifetime $\tau_{\mathrm{R}} \approx 35 \mu \mathrm{s}$. The radiative decays of $\mid \mathrm{e}, 1>$ and $\mid \mathrm{e}, 2>$ differ in the degree of polarization of the fluorescence light. The transition $|\mathrm{e}, 1>\rightarrow| \mathrm{e}, 2>$ preserves the angular momentum couplings, the g-factor, and even the Zeeman coherence in the magnetic sublevels of the hfs-level. These results imply that the transition $|\mathrm{e}, 1>\rightarrow| \mathrm{e}, 2>$ is a change of the vibronic state of the molecule, whereas the rotationspin state describing the rotation and its coupling to electron spin and nuclear spin is obviously not affected by this transition. The time constant $\tau_{0}$ agrees well with the lifetime $\tau_{\text {in }}$ evaluated from the integrated absorption coefficient $[18,20]$. Thus, the experimental results also provide new insight into the lifetime problem of $\mathrm{NO}_{2}$ according to which the radiative lifetime $\tau_{\mathrm{R}}$ is much longer than the lifetime $\tau_{\text {in }}$. According to theory $\tau_{\text {in }}$ is the characteristic time of the mechanism that deactivates the upper state of the optical transition [23]. The various experiments add up to a consistent body of results.

The model of the space-coupled molecule introduced in Sect. 1 and Ref.[11] gives a consistent description of all experimental results. This model implies that the collision-free $\mathrm{NO}_{2}$ is not isolated. It is always coupled to the extra compactified dimensions of space via the gravitational interaction in the extra compactified dimensions. The model explains the inversion effect and the measurements of the narrow and the broad Hanle signal associated with the states $\mid \mathrm{e}, 1>$ and $|\mathrm{e}, 2\rangle$ and with the time constants $\tau_{R}$ and $\tau_{0}$ respectively. The experimental results reported in Sects. 2-5 reveal that the molecule may undergo many cycles of absorption and stimulated emission in the optical transition $|\mathrm{g}, 1>\leftrightarrow| \mathrm{e}, 1>$ before it evolves into $\mid \mathrm{e}, 2>$ via the transition $|\mathrm{e}, 1>\rightarrow| \mathrm{e}, 2>$. This is a characteristic feature of the optical excitation of $\mid e, 1>$. The many cycles of absorption and stimulated emission in the excitation process explain the inversion effect, the minimum of the fluorescence intensity $\mathrm{F}$ in Fig. 3 (Sect. 2) versus the divergence of the light beam, and the strong enhanced absorption resonances reported in Sect. 5. These cycles of absorption and stimulated emission in the excitation process indicate that the process causing the nonradiative transition $|\mathrm{e}, 1>\rightarrow| \mathrm{e}, 2>$ does not affect the optical coherence in the excitation process. This result supports strongly a non-electromagnetic inter- action as source for the perturbation acting on the vibrational motion in $\mid \mathrm{e}, 1>$ and causing the transition $|\mathrm{e}, 1>\rightarrow| \mathrm{e}, 2>$, because an electromagnetic interaction perturbs in general more frequently the phase of a molecular system than the population of a molecular level [49].

An irreversible non-radiative transition in the collisionfree $\mathrm{NO}_{2}$ is not in agreement with the presently accepted model for $\mathrm{NO}_{2}$ [19]. The model of interelectronic level mixing discussed in Sect. 1 assumes that the vibronic coupling of the low vibrational levels of the state $\mathrm{A}^{2} \mathrm{~B}_{2}$ with high vibrational levels of the state $\mathrm{X}^{2} \mathrm{~A}_{1}$ is a reversible process such that these coupled states are the expected eigenstates of the molecule. The unusual result $\tau_{R}>>\tau_{\text {in }}$ ("anomalously long radiative lifetime") is explained by the assumption that a vibrational level of the state $\mathrm{A}^{2} \mathrm{~B}_{2}$ (parent state) is mixed by vibronic coupling with many high lying vibrational levels of the electronic ground state. This mixing dilutes the radiative decay rate by a factor depending on the number of levels of the electronic ground state coupled with the parent state [19]. The model is based on broad band optical excitation of the molecule by a short light pulse and makes no predictions for narrow band optical excitation. We note that our experiments yield $\tau_{0}$ and $\tau_{R}$ using optical excitation of the molecule with a spectral width of the exciting laser light of less than 1 $\mathrm{MHz}$ essentially restricted by transit time broadening of the molecules travelling through the light beam. The model of interelectronic level mixing is not in agreement with the inversion effect and not able to explain two Hanle signals in the fluorescence light one with the coherence decay time $\tau_{0}$ and another one with the coherence decay time $\tau_{R}$. Consequently, the model of interelectronic level mixing is not in agreement with the experimental results.

The model of the space-coupled molecule suggests that the time constant $\tau_{0} \approx 3 \mu \mathrm{s}$ is not a property of the molecule but a property of space. Measurements on $\mathrm{NO}_{2}$ at the wavelengths $\lambda_{\mathrm{ex}} \approx 593 \mathrm{~nm}$ and $\lambda_{\mathrm{ex}} \approx 514 \mathrm{~nm}$ reveal the same values for $\tau_{0}$. Moreover, data from other molecules are also available if we identify $\tau_{0}$ with $\tau_{\text {in }}$. As for $\mathrm{NO}_{2}$, the molecules $\mathrm{SO}_{2}$ and $\mathrm{CS}_{2}$ reveal $\tau_{\mathrm{R}}>>\tau_{\text {in }}$ with $\tau_{\text {in }} \approx 3 \mu$ s for $\mathrm{CS}_{2}$ and $\tau_{\text {in }} \approx 0.2 \mu$ s for $\mathrm{SO}_{2}$ [18]. The result for $\mathrm{SO}_{2}$ differs strongly from the value for $\tau_{\text {in }}$ on $\mathrm{NO}_{2}$, but the values for $\tau_{\text {in }}$ varied strongly in the past. First measurements of $\tau_{\text {in }}$ on $\mathrm{NO}_{2}$ resulted in $\tau_{\text {in }} \approx 0.3 \mu \mathrm{s}$ [51]. Later experiments showed that $\tau_{\text {in }} \approx 3 \mu$ s is a more realistic value [20]. The measurements in Sect. 5 (e.g. the $\sigma$-resonance) suggest that reliable absorption measurements should be made at zero magnetic field (compensation of the earth magnetic field).

In conclusion, the experimental results reported in this paper disagree with the presently accepted model of interelectronic level mixing used to describe the molecular dynamics in $\mathrm{NO}_{2}$. On the other hand, the model of the space-coupled molecule considered in this paper describes these experimental results on $\mathrm{NO}_{2}$ in all details. Furthermore, we see no other potential explanation of these results presently. Therefore, we suggest 
ascribing these experimental results to an effect of the extra compactified dimensions of space in the molecule $\mathrm{NO}_{2}$.

Acknowledgements This work is dedicated to my friend and colleague Dr. Franciszek Bylicki, Torun, Poland, who died in 2016. He contributed much to the experimental work reported here.

Funding Open Access funding enabled and organized by Projekt DEAL.

Data Availability Statement This manuscript has associated data in a data repository. [Authors' comment: All data analysed during this study are included in this published article or are given in the references cited by this article.]

Open Access This article is licensed under a Creative Commons Attribution 4.0 International License, which permits use, sharing, adaptation, distribution and reproduction in any medium or format, as long as you give appropriate credit to the original author(s) and the source, provide a link to the Creative Commons licence, and indicate if changes were made. The images or other third party material in this article are included in the article's Creative Commons licence, unless indicated otherwise in a credit line to the material. If material is not included in the article's Creative Commons licence and your intended use is not permitted by statutory regulation or exceeds the permitted use, you will need to obtain permission directly from the copyright holder. To view a copy of this licence, visit http://creativecomm ons.org/licenses/by/4.0/.

\section{Appendix A}

In Ref. [46] we introduce a phenomenological model of the inversion effect and of the broad rf-resonance based on the Eqs. 1 in Sect. 1, and we rationalize the transition $|\mathrm{e}, 1>\rightarrow| \mathrm{e}, 2>$ by postulating, as in hiddenvariables theories, an additional variable to the wellknown set of quantum numbers which are commonly used to describe a pure quantum state of the molecule. In the following we use the equations of this model, but we identify the "hidden" variable with the gravitational coupling of the molecule to the extra compactified dimensions of space as proposed in the model of the space-coupled molecule. With this identification the equations of motion derived in Ref. [46] apply also to the space-coupled molecule. We introduce $\mid \mathrm{a}>=$ $|\mathrm{g}, 1>,| \mathrm{b}>=\mid \mathrm{e}, 1>$, and $|\mathrm{c}>=| \mathrm{e}, 2>$, and the equations of motion of an ensemble of molecules represented by the Hamiltonian $\mathrm{H}=\mathrm{H}_{0}+\mathrm{V}+\mathrm{G}+\underline{\mathrm{B}}$ in the subspace of states $\mid u>=\{|a>| b>,, \mid c>\}$ with the magnetic sublevels $\mid \mathrm{u}, \mathrm{m}>=\{|\mathrm{a}, \mathrm{m}>,| \mathrm{b}, \mathrm{m}>, \mid \mathrm{c}, \mathrm{m}>\}$. We assume that the states $\mid \mathrm{u}>$ are eigenstates of $\mathrm{H}_{0}$ with $\mathrm{H}_{0}\left|\mathrm{u}>=\mathrm{E}_{\mathrm{u}}\right| \mathrm{u}>$ and $\mathrm{E}_{\mathrm{a}}<\mathrm{E}_{\mathrm{b}}=\mathrm{E}_{\mathrm{c}}$. Here $\mathrm{H}_{0}$ is the Hamiltonian of the space-coupled molecule and $\mathrm{V}$ represents an asymmetric perturbation acting on the non-rigid compactification space and causing the irreversible transition $|\mathrm{b}>\rightarrow| \mathrm{c}>$ associated with the time constant $\tau_{0}$. We assume that $\mathrm{V}$ is effective in $|\mathrm{b}\rangle$ but not in $\mid c>$. G represents the interaction with the light field of the laser inducing the optical transition $|\mathrm{a}\rangle \leftrightarrow|\mathrm{b}\rangle$, and $\underline{\mathrm{B}}$ represents the interaction with the static magnetic field $\mathrm{B}$ and the rf-field $\mathrm{B}_{1}$. The light field of the laser is introduced as classical field and the effect of spontaneous emission is described phenomenologically. Following Ref.[46], we introduce the density matrix elements $\rho_{u v}^{m n}=\langle u, m|\rho| \nu, n\rangle$ of the ensemble of molecules in the subspace of states $\{|\mathrm{u}, \mathrm{m}>,| \mathrm{v}, \mathrm{n}>\}$ with $\mathrm{u}, \mathrm{v}=\mathrm{a}, \mathrm{b}, \mathrm{c}$ and the magnetic quantum numbers $\mathrm{m}, \mathrm{n}$. The equations of motion are Eqs. A1 - A6.

$$
\begin{aligned}
& \dot{\rho}_{a a}^{m n}=-Z_{a a}^{m n} \rho_{a a}^{m n}-i \Sigma_{r}\left(G_{a b}^{m r} \rho_{b a}^{r n}-\rho_{a b}^{m r} G_{b a}^{r n}\right) \\
& \quad+\gamma_{a a} \rho^{0} \delta_{m, n}+W_{a a}^{m n} \\
& \dot{\rho}_{b b}^{m n}=-Z_{b b}^{m n} \rho_{b b}^{m n}-i \Sigma_{r}\left(G_{b a}^{m r} \rho_{a b}^{r n}-\rho_{b a}^{m r} G_{a b}^{r n}\right) \\
& \quad-i\left(V_{b c} \rho_{c b}^{m n}-\rho_{b c}^{m n} V_{c b}\right)+W_{b b}^{m n} \\
& \dot{\rho}_{c c}^{m n}=-Z_{c c}^{m n} \rho_{c c}^{m n}-i\left(V_{c b} \rho_{b c}^{m n}-\rho_{c b}^{m n} V_{b c}\right)+W_{c c}^{m n}(\mathrm{~A} 2 \\
& \dot{\rho}_{a b}^{m n}=-Z_{a b}^{m n} \rho_{a b}^{m n}-i \Sigma_{r}\left(G_{a b}^{m r} \rho_{b b}^{r n}-\rho_{a a}^{m r} G_{a b}^{r n}\right)+W_{a b}^{m n} \\
& \dot{\rho}_{b c}^{m n}=-Z_{b c}^{m n} \rho_{b c}^{m n}-i \Sigma_{r}\left(G_{b a}^{m r} \rho_{a c}^{r n}\right)+i V_{b c} \rho_{b b}^{m n}+W_{b c}^{m n} \\
& \dot{\rho}_{a c}^{m n}=-Z_{a c}^{m n} \rho_{a c}^{m n}-i \Sigma_{r}\left(G_{a b}^{m r} \rho_{b c}^{r n}\right)+W_{a c}^{m n} \\
& Z_{\mathrm{uv}}^{m n}=\mathrm{i}\left(\hbar^{-1}\right)\left(\mathrm{E}_{\mathrm{u}}-\mathrm{E}_{\mathrm{v}}\right)+\mathrm{i}\left(\mathrm{m} \omega_{\mathrm{u}}-\mathrm{n} \omega_{\mathrm{v}}\right)+\Gamma_{\mathrm{uv}} \\
& W_{u v}^{m n}=-i \Sigma_{r}\left(B_{\mathrm{uu}}^{m r} \rho_{\mathrm{uv}}^{r n}-\rho_{\mathrm{uv}}^{m r} B_{\mathrm{vv}}^{r n}\right) \\
& <\mathrm{u}, \mathrm{m}\left|\mathrm{B}+\mathrm{B}_{1}\right| \mathrm{v}, \mathrm{n}>=\hbar \mathrm{m} \omega_{\mathrm{u}} \delta_{\mathrm{m}, \mathrm{n}} \delta_{\mathrm{u}, \mathrm{v}}+\hbar \mathrm{B}_{\mathrm{uv}}^{m n} \delta_{\mathrm{u}, \mathrm{v}}
\end{aligned}
$$

Eqs. A7-A9 define components of the equations of motion, the eigen-energies $\mathrm{E}_{\mathrm{u}}$ and $\mathrm{E}_{\mathrm{v}}$ of $\mathrm{H}_{0}$ (see above), the magnetic field interaction matrix elements $<\mathrm{u}, \mathrm{m}\left|\mathrm{B}+\mathrm{B}_{1}\right| \mathrm{v}, \mathrm{n}>$ with the static magnetic field $\mathrm{B}$ parallel and the rf-field $\mathrm{B}_{1}$ perpendicular to the quantization axis, and the phenomenologically introduced rate constants $\Gamma_{\text {uv }}$ representing the effects of the transition $|\mathrm{b}>\rightarrow| \mathrm{c}>$ and of spontaneous emission e.g. on the coherence decay of the off-diagonal density matrix elements. The term $\gamma_{a a} \rho^{0} \delta_{\mathrm{m}, \mathrm{n}}$ in Eq. A1 represents the initial state of the molecule before interaction with the light beam. In these equations $G_{a b}^{m r}$ represents the interaction with the optical field inducing the transition $|\mathrm{a}>\leftrightarrow| \mathrm{b}>$. We assume $<\mathrm{u}, \mathrm{m}|\mathrm{V}| \mathrm{v}, \mathrm{n}>=\hbar \mathrm{V}_{\mathrm{uv}} \delta_{\mathrm{m}, \mathrm{n}}$ and $\mathrm{V}_{\mathrm{uv}}=0$ except $\mathrm{V}_{\mathrm{bc}}=\mathrm{V}_{\mathrm{cb}} *=$ constant $\neq 0$. We propose an irreversible evolution $|\mathrm{b}>\rightarrow| \mathrm{c}\rangle$ and describe this by postulating $\mathrm{V}_{\mathrm{bc}} \rho_{c c}^{m n} \equiv 0$ in Eq. A5 based on the argument that $\mathrm{V}$ is not effective in $|\mathrm{c}>=| \mathrm{e}, 2>$.

In Ref. [46] we derive from Eqs. A1- A9 the rate equations, Eqs. 7a - 7d in Sect. 3, describing the inversion effect. We introduce

$$
\begin{aligned}
& \mathrm{a}_{\mathrm{m}}=\rho_{a a}^{m m}, \mathrm{~b}_{\mathrm{m}}=\rho_{b b}^{m m}, \mathrm{c}_{\mathrm{m}}=\rho_{c c}^{m m}, \\
& \gamma_{m}=\left|G_{a b}^{m m}\right|^{2}\left(\Gamma_{a c}+b_{L}\right)^{-1}
\end{aligned}
$$


assuming that the laser light is tuned to resonance. The decay rate for the nonradiative transition $\mid \mathrm{b}>\rightarrow$ $\mid c>$ is

$$
\begin{aligned}
\lambda_{m} & =\frac{2\left|V_{b c}\right|^{2}}{\Gamma_{b c}+\gamma_{m}\left[1-\exp \left(-\Gamma_{b c} T_{L}\right)\right]} \\
& =\frac{1}{\tau_{0}} \frac{1}{1+\gamma_{m} \tau_{0}\left[1-\exp \left(-T_{L} / \tau_{0}\right)\right]}
\end{aligned}
$$

Here we use $\Gamma_{b c}=1 / \tau_{0}$ and assume $\lambda_{m}=1 / \tau_{0}$ for $\Gamma_{b c} T_{\mathrm{L}} \ll 1$. This gives $2\left|V_{b c}\right|^{2} \tau_{0}^{2}=1$.

Also, in Ref. [46], we calculate the broad rf-resonance (Eqs. 10a-10b in Sect. 5). We assume that the fluorescence intensity $\mathrm{F}$ is in good approximation proportional to the occupation probability $\Sigma_{\mathrm{m}} \mathrm{c}_{\mathrm{m}}$ of $|\mathrm{c}\rangle$, because the fluorescence intensity of $\mid \mathrm{b}>$ is much smaller $(<1 / 10)$ than the fluorescence intensity of $|c\rangle$. The calculation of $\Sigma_{m} c_{m}$ is performed using an approximation for low power of the rf-field and of the light intensity. The result is (see Eqs. 4.5-4.8 of Ref. [46])

$$
\begin{aligned}
\mathrm{F} \sim \Sigma_{\mathrm{m}} \mathrm{c}_{\mathrm{m}} & =\Sigma_{\mathrm{m}} \mathrm{b}_{\mathrm{m}}\left(\tau_{\mathrm{R}} \lambda_{\mathrm{ms}}\right) \\
\lambda_{m s} & =\frac{\left|V_{b c}\right|^{2}}{\Gamma_{b c}+\gamma_{m}\left[1-\exp \left(-\Gamma_{b c} T_{L}\right)\right]\left(1-W_{m}\right)}+c . c . \\
W_{m} & =\frac{1}{\left(\Gamma_{a c}+b_{L}\right)} \sum_{r}\left[K_{r}(a)+K_{r}(b)\right] \\
K_{r}(a) & =\frac{\left|B_{a a}^{m m+r}\right|^{2}}{\left[i r\left(\omega_{a}-\omega_{r f}\right)+\left(\Gamma_{a c}+b_{L}\right)\right]}
\end{aligned}
$$

The rf-field appears only in $\mathrm{W}_{\mathrm{m}}$. Without an rf-field we have $\mathrm{W}_{\mathrm{m}}=0$ and $\lambda_{\mathrm{ms}}=\lambda_{\mathrm{m}}$. In Eq. A12 we assume $\left|\mathrm{W}_{\mathrm{m}}\right|<1$ because of the low rf-power approximation. The quantity $\mathrm{W}_{\mathrm{m}}$ comprises two terms representing rf-resonances in the lower state $\left(K_{r}(a)\right)$ and in the upper state $\left(K_{r}(b)\right)$. Both terms have the same form. In $K_{r}(a)$, we neglected the spectral width of the rf-field which is much smaller than the spectral width $\mathrm{b}_{\mathrm{L}}$ of the light field. We assume $\mathrm{r}= \pm 1$ because only rf-field transitions between neighbouring sublevels are important in the low rf-power approximation. For the comparison with the experiment, we evaluate the quantity $\mathrm{S}_{\mathrm{b}}=\left(\mathrm{F}-\mathrm{F}_{0}\right) / \mathrm{F}_{0}$ where $\mathrm{F}_{0}=\mathrm{F}$ for $\mathrm{W}_{\mathrm{m}}=0$ using an approximation for low light intensity. The details of the approximation are described in Refs. [35,46]. The result is Eqs. 10 in Sect. 5. We note here that Eq. A3 is correcting a misprint in Eq. 2.6 of Ref. [46]. Another misprint is $R_{u v}^{m n}$ instead of $B_{u v}^{m n}$ in Eqs. 4.3 and 4.4 of Ref. [46].

\section{References}

1. K. Becker, M. Becker, J.H. Schwarz, String Theory and M-theory: A Modern Introduction (Cambridge University Press, Cambridge, 2007)
2. B. Zwiebach, A First Course in String Theory, 2nd edn. (Cambridge University Press, Cambridge, 2009)

3. N. Arkani-Hamed, S. Dimopoulos, G.R. Dvali, Phys. Lett. B 429, 263 (1998)

4. I. Antoniadis, N. Arkani-Hamed, S. Dimopoulos, G.R. Dvali, Phys. Lett. B 436, 257 (1998)

5. L. Randall, R. Sundrum, Phys. Rev. Lett. 83, 3370 (1999)

6. K.R. Dienes, E. Dudas, T. Gherghetta, Nucl. Phys. B 537, 47 (1999)

7. L. Randall, M.D. Schwartz, Phys. Rev. Lett. 88, 081801 (2002)

8. H.C. Cheng, K.T. Matchev, M. Schmaltz, Phys. Rev. D 66, 036005 (2002)

9. E.J. Salumbides, A.N. Schellekens, B. Gato-Rivera, W. Ubachs, New J. Phys. 17, 033015 (2015)

10. W. Ubachs, J.C.J. Koelemeij, K.S.E. Eikema, E.J. Salumbides, J. Mol. Spectrosc. 320, 1 (2016)

11. H.G. Weber, J. Phys. Commun. 3, 101001 (2019)

12. S. Mahapatra, H. Köppel, L.S. Cederbaum, P. Stampfuss, W. Wenzel, Chem. Phys. 259, 211 (2000)

13. W. Domcke, D.R. Yarkony, H. Köppel, Eds., Conical Intersections: Electronic Structure, Dynamics and Spectroscopy, Vol. 15, Advanced Series in Physical Chemistry (World Scientific, Singapore, 2004)

14. M. Sanrey, M. Joyeux, J. Chem. Phys. 125, 014304 (2006)

15. Y. Arasaki, K. Takatsuka, K. Wang, V. McKoy, J. Chem. Phys. 132, 124307 (2010)

16. H.J. Wörner et al., Science 334, 208 (2011)

17. D.K. Hsu, D.L. Monts, R.N. Zare, Spectral Atlas of Nitrogen Dioxide 5530 to 6480 AA (Academic Press, New York, 1978)

18. A.E. Douglas, J. Chem. Phys. 45, 1007 (1966)

19. M. Bixon, J. Jortner, J. Chem. Phys. 50, 3284 (1969)

20. V.M. Donnelly, F. Kaufman, J. Chem. Phys. 69, 1456 (1978)

21. C.G. Stevens, R.N. Zare, J. Mol. Spectrosc. 56, 167 (1975)

22. T. Tanaka, R.W. Field, D.O. Harris, J. Mol. Spectrosc. 56, 188 (1975)

23. S.J. Strickler, R.A. Berg, J. Chem. Phys. 37, 814 (1962)

24. C.H. Chen, S.P. Kramer, D.W. Clark, M.G. Payne, Chem. Lett. 65, 419 (1979)

25. O. Cheshnovsky, A. Amirav, Chem. Phys. Lett. 109, 368 (1984)

26. F. Bylicki, H.G. Weber, G. Persch, W. Demtröder, J. Chem. Phys. 88, 3532 (1988)

27. H.G. Weber, F. Bylicki, G. Miksch, Phys. Rev. A 30, 270 (1984)

28. F. Bylicki, J. Phys. B At. Mol. Opt. Phys. 27, 283 (1994)

29. F. Bylicki, H.G. Weber, H. Zscheeg, M. Arnold, J. Chem. Phys. 80, 1791 (1984)

30. F. Bylicki, H.G. Weber, J. Chem. Phys. 78, 2899 (1983)

31. P.J. Brucat, R.N. Zare, J. Chem. Phys. 78, 100 (1983)

32. H.G. Weber, Phys. Lett. A 129, 355 (1988)

33. R. Solarz, D.H. Jevy, J. Chem. Phys. 60, 842 (1974)

34. H.G. Weber, F. Bylicki, Chem. Phys. 116, 133 (1987)

35. H.G. Weber, G. Miksch, Phys. Rev. A 31, 1477 (1985)

36. H.G. Weber in G. Moruzzi, F. Strumia, The Hanle Effect and Level-Crossing Spectroscopy (Plenum Press, New York, 1991) 
37. H.G. Weber, Z. Phys. D. Atoms. Mol. Clust. 1, 403 (1986)

38. S. Sardar, S. Mukherjee, A.K. Paul, S. Adhikari, Chem. Phys. 416, 11 (2013)

39. H. Ruf et al., J. Chem. Phys. 137, 224303 (2012)

40. J. Brossel, F. Bitter, Phys. Rev. 86, 308 (1952)

41. G. Moruzzi, F. Strumia, The Hanle Effect and LevelCrossing Spectroscopy (Plenum Press, New York, 1991)

42. H.G. Weber, F. Bylicki, Z. Phys, D-Atoms, Molecules and Clusters 8, 279 (1988)

43. I.R. Bonilla, W. Demtröder, Chem. Phys. Lett. 53, 223 (1978)

44. H. Figger, D.L. Monts, R.N. Zare, J. Mol. Spectry. 68, 388 (1977)
45. F. Bylicki, H.G. Weber, Phys. Lett. A 100, 182 (1984)

46. H.G. Weber, Phys. Rev. A 31, 1488 (1985)

47. R.N. Zare, J. Chem. Phys. 45, 4510 (1966)

48. G. Herzberg, Molecular Spectra and Molecular Structure, vol. III (van Nostrand Reinhold Co., New York, 1966)

49. K. Shimoda, in High-Resolution Laser Spectroscopy Sec.2, edited by K. Shimoda (Springer-Verlag, Berlin, 1976)

50. D.K.C. MacDonald, Noise and Fluctuations: An Introduction (Wiley, New York, 1962)

51. D. Neuberger, A.B.F. Duncan, J. Chem. Phys. 22, 1693 (1954) 